\title{
Optimal Energy-Efficient Policies for Data Centers through Sensitivity-Based Optimization
}

\author{
Jing-Yu Ma*, Li Xia ${ }^{\dagger}$ Quan-Lin Li
}

\begin{abstract}
In this paper, we propose a novel dynamic decision method by applying the sensitivity-based optimization theory to find the optimal energy-efficient policy of a data center with two groups of heterogeneous servers. Servers in Group 1 always work at high energy consumption, while servers in Group 2 may either work at high energy consumption or sleep at low energy consumption. An energy-efficient control policy determines the switch between work and sleep states of servers in Group 2 in a dynamic way. Since servers in Group 1 are always working with high priority to jobs, a transfer rule is proposed to migrate the jobs in Group 2 to idle servers in Group 1. To find the optimal energy-efficient policy, we set up a policy-based Poisson equation, and provide explicit expressions for its unique solution of performance potentials by means of the RG-factorization. Based on this, we characterize monotonicity and optimality of the long-run average profit with respect to the policies under different service prices. We prove that the bang-bang control is always optimal for this optimization problem, i.e., we should either keep all servers sleep or turn on the servers such that the number of working servers equals that of waiting jobs in Group 2. As an easy adoption of policy forms, we further study the threshold-type policy and obtain a necessary condition of the optimal threshold policy. We hope the methodology and results derived in this paper can shed light to the study of more general energy-efficient data centers.
\end{abstract}

Keywords: Queueing; Data center; Energy-efficient policies; Sensitivity-based optimization; Markov decision process.

\footnotetext{
${ }^{*}$ Jing-Yu Ma and Quan-Lin Li are both with the School of Economics and Management Sciences, Yanshan University, Qinhuangdao 066004, China (e-mail: mjy0501@126.com, liquanlin@tsinghua.edu.cn).

${ }^{\dagger} \mathrm{Li} \mathrm{Xia}$ is with the Center for Intelligent and Networked Systems (CFINS), Department of Automation, TNList, Tsinghua University, Beijing 100084, China (e-mail: xial@tsinghua.edu.cn).
} 


\section{Introduction}

Data centers have become a core part of the IT infrastructure for Internet service. Typically, hundreds of thousands of servers are deployed in a data center to provide ubiquitous computing environments. Tremendous energy consumption becomes a significant operation expense of data centers. In 2014, the electricity consumption of data centers in the USA estimated 70 billion KWh, accounted for $2 \%$ of the national electricity consumption [43. The data centers in the USA are expected to consume energy $140 \mathrm{TWh}$ and spend $\$ 13$ billion energy bills by 2020 [37, while these figures in Europe will reach 104 TWh and $\$ 9.6$ billion [7]. The energy consumption of data centers consists of three main parts: servers, networks and cooling, while servers are the major one. It is estimated that servers consume around $70 \%$ of the total energy consumption in a data center with tiered architectures [26]. On the other hand, reducing the energy consumption of servers also can help reduce the energy consumption of networking and cooling. Therefore, energy-efficient scheduling of servers is of significance for the energy management of data centers.

During the last two decades, considerable attention has been paid to studying the energy efficiency of data centers. An early interesting observation by Barroso and Hölzle [1] demonstrated that a lot of data centers were designed to be able to handle the peak loads effectively, but it directly caused that a significant number of servers (about 20\%) are often idle in the off-peak period. Although the idle servers do not provide any service, they still consume a notable amount of energy. Therefore, it is necessary to design an energy-efficient mechanism for effectively saving energy of idle servers. Previous studies demonstrate that a potential power cutting could be as remarkable as $40 \%$ [4]. For this purpose, a key technique, called an energy-efficient state 'sleep' or 'off', was introduced to save energy for idle servers. See Gandhi et al. [14] and Kuehn and Mashaly [28] for more interpretations. In this case, some queueing models either with server energy-efficient states (e.g., work, idle, sleep, and off) or with server control policies (e.g., vacation, setup, and $N$-policy) were developed in the study of energy-efficient data centers. Queueing theory and Markov (reward or decision) processes become two useful mathematical tools in analyzing energy-efficient data centers. See Gandhi [9] and Li et al. [31] for more details.

Few available studies have applied queueing theory and Markov processes to performance analysis and optimization of energy-efficient data centers. Important examples in the recent literature are remarked as follows. Gandhi et al. [11] considered a data cen- 
ter with multiple identical servers, the states of which include work, idle, sleep and off, and their energy consumption have a decreasing order. One crucial technique given in Gandhi et al. [11,13] was to develop some interesting queueing models, for example, the $\mathrm{M} / \mathrm{M} / \mathrm{k}$ queue with setup times. Since then, some multi-server queues have received attention (for example, queues with server vacations, queues with either local setup times or $N$-policy), and they were successfully applied to energy-efficient management of data centers. Readers may refer to recent publications for more details, among which are Mazzucco et al. [34, Schwartz et al. 42, Gandhi and Harchol-Balter [12, Gandhi et al. [10], Maccio and Down [33], Phung-Duc [38, Chen et al. [6], and Li et al. [31].

In the study of energy-efficient data centers, it is a key to develop effective optimal methods and dynamic control techniques in data centers. So far, there have been two classes of optimal methods applied to the analysis of energy-efficient data centers. The first class is regarded as 'static optimization' with two basic steps. Step one is to set up performance cost (i.e., a suitable performance-energy tradeoff) of a data center, where the performance cost can be expressed by means of queueing indexes of the data center. Step two is to optimize the performance cost with respect to some key parameters of the data center by using, such as, linear programming, nonlinear programming, integer programming, and bilevel programming. The second class is viewed as 'dynamic optimization' in which Markov decision processes or stochastic network optimization are applied to energyefficient management of data centers, e.g., see Benini et al. 2] and Yao et al. [57] for more details.

For the static optimization, some available works have been successfully conducted according to two key points: The first key point is to emphasize how to construct a suitable utility function for the performance-energy tradeoff, which needs to synchronously optimize several different performance measures, for example, reducing energy consumption, reducing system response time, and improving quality of service. The second key point is to minimize performance cost with respect to some crucial parameters of data centers by means of, such as linear programming and nonlinear programming. On such a research line, Gandhi et al. [1] recalled two classes of performance-energy tradeoffs: (a) ERWS, the weighted sum $\beta_{1}[R]+\beta_{2}[E]$ of the mean response time $[R]$ and the mean power cost $[E]$, where $\beta_{1}, \beta_{2} \geq 0$ are weighted coefficients; and (b) ERP, the product $[R][E]$ of the mean response time and the mean power cost. For the ERP, Gandhi et al. [1] first described the data center as a queue to compute the two mean values $[R]$ and $[E]$, and 
then provided an optimization method to minimize the ERP. Also, they further analyzed optimality or near-optimality of several different energy-efficient policies. In addition, Gandhi [9] gave some extended results and a systematical summarization with respect to minimizing the ERP. Maccio and Down [33] generalized the ERP by Gandhi [11] to a more general performance cost function as follows.

$$
f(\beta, w)=\sum_{i=1}^{M} \beta_{i}([R])^{w_{R, i}}([E])^{w_{E, i}}([C])^{w_{C, i}},
$$

where $[C]$ is the expected cycle rate, and $\beta_{i}, w_{R, i}, w_{E, i}$ and $w_{C, i}$ for $1 \leq i \leq M$ are nonnegative weighted coefficients, $\beta=\left(\beta_{i}: 1 \leq i \leq M\right)$ and $w=\left(w_{R, i}, w_{E, i}, w_{C, i}: 1 \leq i \leq M\right)$. They used the queueing models to compute the three mean values $[R],[E]$ and $[C]$, and then provided some discussion on the optimality of cost function $f(\beta, w)$. Gebrehiwot et al. [15] made another interesting generalization of the ERP and ERWS by Gandhi [11] through introducing the multiple intermediate sleep states. Under more general assumptions with general service and setup times, they computed the two mean values $[R]$ and $[E]$ by means of some queueing insensitivity, and then discussed the optimality of the ERP and ERWS. Further, Gebrehiwot et al. [16, 17] generalized the FCFS queueing results of the data center with multiple intermediate sleep states to the processor-sharing discipline and the shortest remaining processing time (SRPT) discipline, respectively. Different from the ERP and ERWS, Mitrani [35,36] considered a data center of $N$ identical servers whose first part contains $m$ servers. The idle or work state of servers is controlled by two different thresholds: an up threshold $U$ and a down threshold $D$. He designed a simple three-layer queue to describe the energy-efficient data center in terms of a new performance cost: $C=c_{1} L+c_{2} S$, where $L$ and $S$ are the average numbers of jobs present and of energy consumption, respectively. He provided expressions for computing the average numbers $L$ and $S$ such that the performance cost $C$ can be optimized with respect to the three parameters $m, U$ and $D$.

However, for the dynamic optimization, little work has been done on applying Markov decision processes to set up optimal dynamic control policies for energy-efficient data centers. In general, such a study is more interesting, difficult and challenging due to the fact that a complicated queueing model with synchronously multiple control objectives (e.g., reducing energy consumption, reducing system response time and guaranteeing quality of service) needs to be synthetically established in a Markov decision process. For a data center with multiple identical servers, Kamitsos et al. [23 25] constructed a discrete-time 
Markov decision process by uniformization and proved that the optimal sleep energyefficient policy is simply hysteretic. Hence, this problem has a double threshold structure by means of the optimal hysteretic policy given in Hipp and Holzbaur [19] and Lu and Serfozo [32]. On the other hand, as some close research to energy-efficient data centers, it is worthwhile to note that the policy optimization and dynamic power management for electronic systems or equipments were developed well by means of Markov decision processes and stochastic network optimization. Important examples include: (a) Discretetime Markov decision processes by Benini et al. 2] and Yang et al. [56]; (b) Continuoustime Markov decision processes by Qiu and Pedram [40] and Qiu et al. [41]; (c) Stochastic network optimization by Yao et al. [57] and Huang and Neely [20]; (d) It has become increasingly important to simplify the method of Markov decision processes such that more complicated stochastic networks can be analyzed effectively. On this research line, eventdriven techniques of Markov decision processes have received high attention for the past one decade. Important examples include the event-driven power management by Šimunić et al. [44, and the event-driven optimization techniques by Becker et al. [3], Cao [5], Koole [27], Engel and Etzion [8], and Xia et al. [52].

The purpose of this paper is to apply the Markov decision processes to set up an optimal dynamic control policy for energy-efficient data centers. To do this, we first apply the sensitivity-based optimization theory in the study of data centers. Note that the sensitivity-based optimization is greatly refined from the Markov decision processes through re-expressing the Poisson equation (corresponding to the Bellman optimality equation) by means of several novel tools, for instance, performance potential and performance difference (see Cao's book [5]). Also, the sensitivity-based optimization theory can be effectively related to the Markov reward processes (e.g., see Li [29] and Li and Cao [30]) so that it is an effective dynamic decision method for performance optimization of many practical Markov systems. The key idea in the sensitivity-based optimization theory is a performance difference equation that can quantify the performance difference of a Markov system under any two different policies. The difference equation gives a clear relation that explains how the system performance is varying with respect to policies. See an excellent book by Cao [5] for more details. So far, the sensitivity-based optimization theory has been applied to performance optimization of queueing systems (or networks). Important examples include an early invited overview by Xia and Cao 48; the MAP/M/1 queue by Xia et al. [50]; the closed queueing networks by Xia and Shihada [54, Xia [46] and 
Xia and Jia [51]; and the open queueing networks by Xia [47] and Xia and Chen [49]. In addition, the sensitivity-based optimization theory was also applied to network energy management, for example, the multi-hop wireless networks by Xia and Shihada [55] and the tandem queues with power constraints by Xia et al. [53].

The main contributions of this paper are twofold. The first one is to apply the sensitivity-based optimization theory to study the optimal energy-efficient policies of data centers for the first time, in which we propose a job transfer rule among the server groups such that the sleep energy-efficient mechanism becomes more effective. Different from previous works in the literature for applying an ordinary Markov decision process to dynamic control of data centers, we propose and develop an easier and more convenient dynamic decision method: sensitivity-based optimization, in the study of energy-efficient data centers. Crucially, this sensitivity-based optimization method may open a new avenue to the optimal energy-efficient policy of more complicated data centers. The second contribution is to characterize the optimal energy-efficient policy of data centers. We set up a policybased Poisson equation and provide explicit expression for its unique solution by means of the RG-factorization. Based on this, we analyze the monotonicity and optimality of the long-run average profit with respect to the energy-efficient policies under some restrained service prices. We obtain the structure of optimal energy-efficient policy. Specifically, we prove that the bang-bang control is optimal for this problem, which significantly reduces the large search space. We also provide an effective way to design and verify the thresholdtype mechanism in practice, which is of great significance to solve the mechanism design problem of energy-efficient data centers. Therefore, the results of this paper give new insights on understanding not only mechanism design of energy-efficient data centers, but also applying the sensitivity-based optimization to dynamic control of data centers. We hope that the methodology and results given in this paper can shed light to the study of more general energy-efficient data centers.

The remainder of this paper is organized as follows. In Section 2, we describe the problem of an energy-efficient data center with two groups of different servers. In Section 3, for the energy-efficient data center, we first establish a policy-based continuous-time birth-death process with finite states. Then we define a suitable reward function with respect to states and policies of the birth-death process. Based on this, we formulate a dynamic optimization problem to find the optimal energy-efficient policy of the data center. In Section 4, we set up a policy-based Poisson equation and provide explicit expression for 
its unique solution by means of the RG-factorization. In Section 5, we define a perturbation realization factor of the policy-based control process of the data center, and analyze how the service price impacts on the perturbation realization factor. In Section 6 , we use the Poisson equation to derive a useful performance difference equation. Based on this, we discuss the monotonicity and optimality of the long-run average profit with respect to the energy-efficient policies, and prove the optimality of the bang-bang control. In Section 7, we use the Poisson equation to further study a class of threshold energy-efficient policies, and obtain the necessary condition of the optimal threshold policy. Finally, we give some discussions and conclude this paper in Section 8.

\section{Problem Description}

In this section, we give a problem description of the energy-efficient problem in data centers. As the large variation of working loads in data centers, it is widely adopted to organize and operate the data center in a multiple-tier architecture such that the on/off scheduling can be performed in different tiers to save energy [26]. In this paper, we study a data center with two groups of heterogeneous servers. There is no waiting room for jobs in the data center, which can be viewed as a loss queue. The assumption of loss queue model is reasonable for data centers and it is also widely used in telephone systems, computer networks, cloud computing, and so on [18,21,45]. In what follows we provide a detailed problem description for the data center.

Server groups: The data center contains two server groups: Groups 1 and 2, each of which is also one of the interactive subsystems of the data center. Groups 1 and 2 contain $n$ and $m$ servers, respectively. Hence the data center contains $n+m$ servers. Servers in the same group are homogeneous and in different groups are heterogeneous. Note that Group 1 is viewed as a base-line group whose servers are always at the work state to guarantee a necessary service capacity in the data center. Each server in Group 1 consumes an amount of energy per unit of time. By contrast, Group 2 is regarded as a reserved group whose servers may either work or sleep so that each of the $m$ servers can switch its state between work and sleep. If one server in Group 2 is at the sleep state, then it consumes a smaller amount of energy to maintain the sleep state.

Power consumption: The power consumption rates (i.e., power consumption per unit of time) for the two groups of servers are described as: $P_{1, W}$ and $P_{2, W}$ for the work 
state in Groups 1 and 2, respectively; and $P_{2, S}$ only for the sleep state in Group 2. We assume that $0<P_{2, S}<P_{2, W}$.

Arrival processes: The arrivals of jobs at the data center are a Poisson process with arrival rate $\lambda$. Each arriving job is assigned to a server of the two groups according to the following allocation rules:

(a) Each server in Group 1 must be fully utilized so that Group 1 provides a priority service over Group 2. If Group 1 has some idle servers, then the arriving job immediately enters the idle server in Group 1 and receives service there. Furthermore, if all the servers of Group 1 are busy but Group 2 has some idle servers, then the arriving job immediately enters an idle server in Group 2 and receives service there.

(b) No waiting room. A job can be served at an idle server or wait at a sleeping server in Group 2. If all the servers of Groups 1 and 2 are occupied, then any arriving job must be lost immediately. Note that each server may contain only one job, hence the total number of jobs in the data center cannot exceed the number $n+m$.

(c) Opportunity cost. Once the data center contains $n+m$ jobs, then any arriving job has to be lost immediately due to no waiting room. This leads to an opportunity cost with respect to the job loss.

Service processes: In Groups 1 and 2, the service times provided by each server are independent and exponential with the service rate $\mu_{1}$ and $\mu_{2}$, respectively. We assume that $\mu_{1} \geq \mu_{2}$ as a fast condition, which makes the prior use of servers in Group 1.

Once a job enters the data center to receive or wait for service, it has to pay a holding cost in Group 1 or Group 2. We assume a so-called cheap condition that the holding cost in Group 1 is always cheaper than that in Group 2. The fast and cheap conditions are intuitive to guarantee the prior use of servers in Group 1. That is, the servers in Group 1 are not only faster but also cheaper than those in Group 2.

If a job finishes its service at a server and leaves the system, then the data center can obtain a fixed service revenue from each served job. The service discipline of each server in the data center is First Come First Serve (FCFS).

Transfer rule: Based on prior use of servers in Group 1, whenever a server in Group 1 becomes idle, an incomplete-service job (if exists) in Group 2 should be transferred to the idle server in Group 1 to save processing time. When a job is transferred from Group 2 to Group 1, the data center needs to pay a transfer cost.

Independence: We assume that all the random variables defined above in the data 
center are independent.

Finally, the data center, together with its operational mode and mathematical notations, are simply depicted in Figure 1.

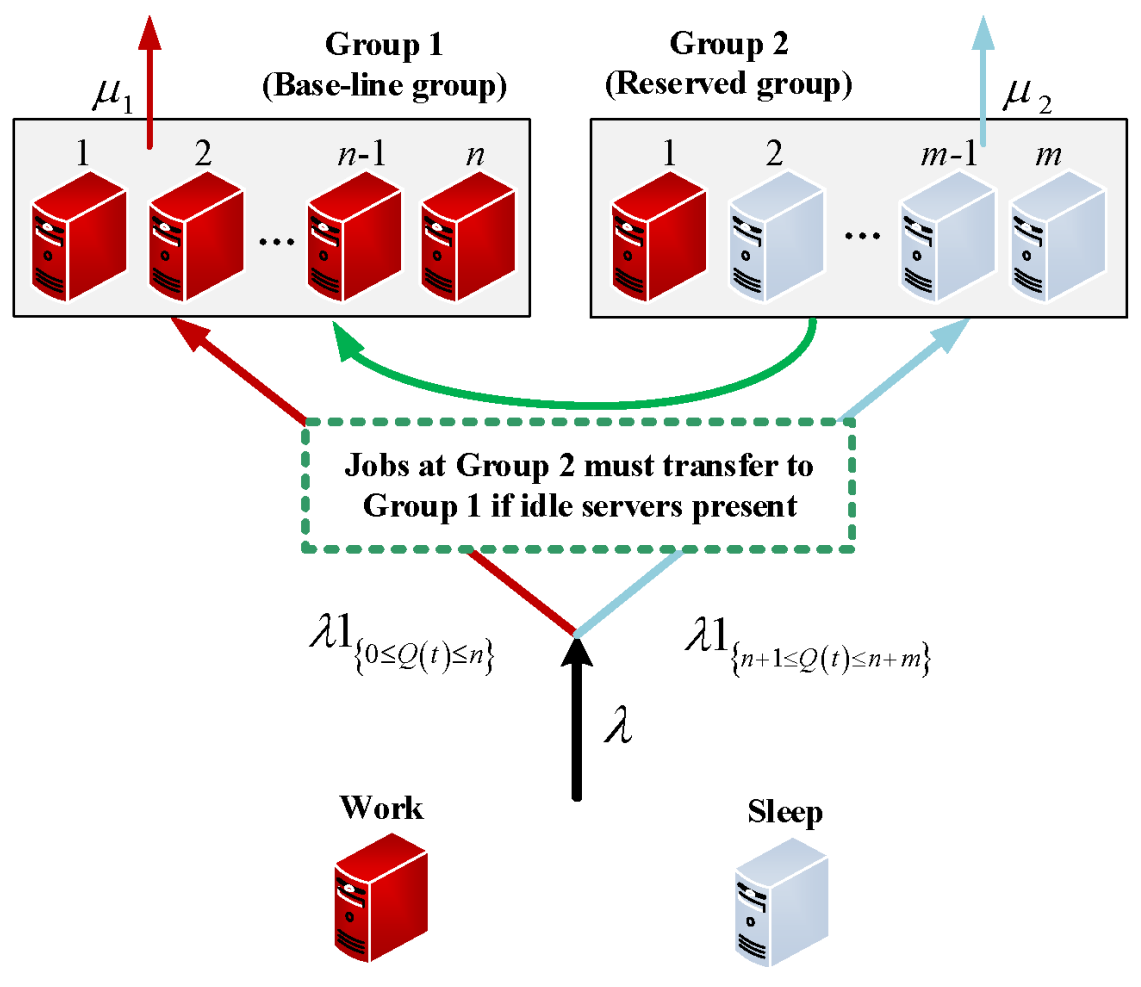

$Q(t):$ The total number of jobs in the two groups at time $t$

Figure 1: Energy-efficient management of data centers.

Remark 1 A further interpretation for the transfer rule: When a job is being served at a server of Group 2, it can be transferred to one idle server of Group 1 and restart its service when an idle server in Group 1 is available. Note that each server in Group 1 is not only faster but also cheaper than that in Group 2, that is, the fast and cheap conditions guarantee that servers in Group 1 have priority than those in Group 2. From the memoryless property of exponential distributions, the restarting service in Group 1 is still faster and cheaper than its original service in Group 2. Therefore, this transfer rule effectively supports energy-efficient management of the data center due to the fact that the servers of Group 1 are fully utilized while servers of Group 2 are kept in sleep state as many as possible. 
Remark 2 Although some authors (e.g., see Gandhi et al. [11], Mitrani [35, 36] and Maccio and Down [33]) analyzed the energy-efficient data center with two groups of servers, where one is the base-line group and the other is the reserved or subsidiary group, all the servers in the two groups given in their papers are assumed to be identical. From such a point of view, it is easy to understand that those queueing models given in their works are the same as only Group 2 of our paper. On the other hand, it is noted that for our queueing model, Group 1 has a large influence on analysis of Group 2 due to introducing new transfer rules. In fact, our queueing model here has been discussed in Li et al. [31] with a more general setting.

\section{Optimization Model Formulation}

In this section, for the energy-efficient data center, we first establish a policy-based continuous-time Markov process, and show that its infinitesimal generator has the simple structure of a birth-death process with finite states. Then, we define a suitable reward function with respect to both states and policies of the birth-death process. Based on this, we set up a dynamic optimization model to deal with the optimal energy-efficient policy of the data center.

In the data center with Group 1 of $n$ servers and Group 2 of $m$ servers, we need to introduce both 'states' and 'policies' to express stochastic dynamics of this data center. Let $I(t)$ and $J(t)$ be the number of jobs in Groups 1 and 2, respectively. Then, $(I(t), J(t))$ is regarded as the state of the data center at time $t$. Let all the cases of state $(I(t), J(t))$ form a state space as follows.

$$
\boldsymbol{\Omega}=\{(0,0),(1,0), \ldots,(n, 0),(n, 1), \ldots,(n, m)\}
$$

Note that such a state $(n-i, j)$ for $1 \leq i \leq n$ and $1 \leq j \leq m$ does not exist according to the transfer rule. However, the policies are defined with a little bit more complication. Let $d_{i, j}$ be the number of servers turned on in Group 2 at the state $(I(t), J(t))=(i, j)$, for $i=0,1, \ldots, n$ and $j=0,1, \ldots, m$. From the problem description in Section 2, it is easy to see that

$$
d_{i, j}= \begin{cases}0, & i=0,1, \ldots, n-1, j=0 \\ 0, & i=n, j=0 \\ 0,1, \ldots, m, & i=n, j=1,2, \ldots, m .\end{cases}
$$


Now, we provide an interpretation for the above expression: If $I(t)=0,1, \ldots, n-1$, then $J(t)=0$ due to the transfer rule. In this case with $i=0,1, \ldots, n, d_{i, 0}$ is taken as zero due to the energy-efficient cause. While once $I(t)=n, J(t)$ may be any element in the set $\{0,1, \ldots, m\}$. In this case with $i=n$ and $j \neq 0, d_{n, j}$ may be taken as any element in the set $\{0,1, \ldots, m\}$.

Corresponding to each case of state $(I(t), J(t))$ at time $t$, we define a time-homogeneous policy as

$$
\boldsymbol{d}=\left(d_{0,0}, d_{1,0}, \ldots, d_{n, 0} ; d_{n, 1}, d_{n, 2}, \ldots, d_{n, m}\right) .
$$

It follows from (1) that

$$
\boldsymbol{d}=\left(0,0, \ldots, 0 ; d_{n, 1}, d_{n, 2}, \ldots, d_{n, m}\right) .
$$

Let all the possible policies $\boldsymbol{d}$ given in (2) compose a policy space as follows.

$$
\mathcal{D}=\left\{\boldsymbol{d}: \boldsymbol{d}=\left(0,0, \ldots, 0 ; d_{n, 1}, d_{n, 2}, \ldots, d_{n, m}\right), d_{i, j} \in\{0,1, \ldots, m\}, \quad(i, j) \in \boldsymbol{\Omega}\right\}
$$

Let $\mathbf{X}^{(\boldsymbol{d})}(t)=(I(t), J(t))^{(\boldsymbol{d})}$ be the system state at time $t$ under any given policy $\boldsymbol{d} \in \mathcal{D}$. Then, $\left\{\mathbf{X}^{(\boldsymbol{d})}(t): t \geq 0\right\}$ is a policy-based continuous-time birth-death process on the state space $\boldsymbol{\Omega}$ whose infinitesimal generator is given by

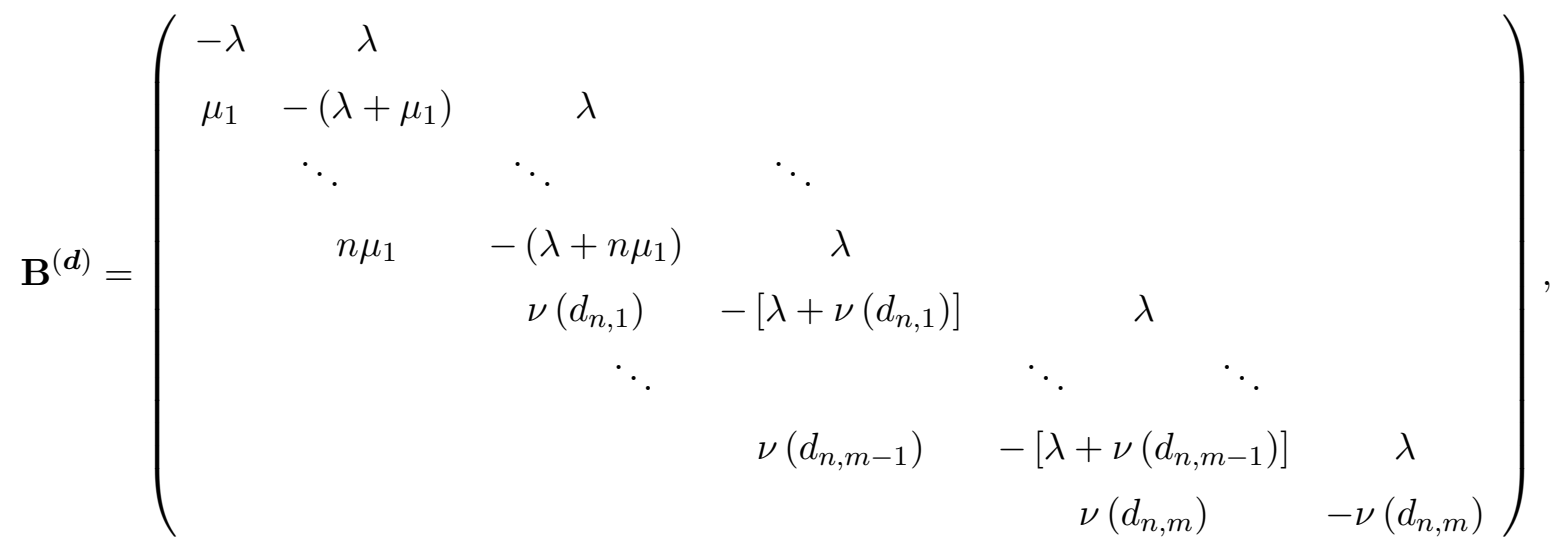

where $\nu\left(d_{n, j}\right)=n \mu_{1}+\left(d_{n, j} \wedge j\right) \mu_{2}$ for $j=1,2, \ldots, m$, and $a \wedge b$ denotes the minimal one between two real numbers $a$ and $b$. Note that $d_{n, j} \geq 0$, it is clear that $\nu\left(d_{n, j}\right) \geq n \mu_{1}>0$. Thus, the birth-death process $\mathbf{B}^{(\boldsymbol{d})}$ must be irreducible, aperiodic and positive recurrent for any given policy $\boldsymbol{d} \in \mathcal{D}$. In this case, we write the stationary probability vector of the Markov process $\left\{\mathbf{X}^{(\boldsymbol{d})}(t): t \geq 0\right\}$ under a policy $\boldsymbol{d} \in \mathcal{D}$.

$$
\boldsymbol{\pi}^{(\boldsymbol{d})}=\left(\pi^{(\boldsymbol{d})}(0,0), \pi^{(\boldsymbol{d})}(1,0), \ldots, \pi^{(\boldsymbol{d})}(n, 0), \pi^{(\boldsymbol{d})}(n, 1), \ldots, \pi^{(\boldsymbol{d})}(n, m)\right) .
$$


Obviously, the stationary probability vector $\boldsymbol{\pi}^{(\boldsymbol{d})}$ is the unique solution to the system of linear equations: $\boldsymbol{\pi}^{(\boldsymbol{d})} \mathbf{B}^{(\boldsymbol{d})}=\mathbf{0}$ and $\boldsymbol{\pi}^{(\boldsymbol{d})} \boldsymbol{e}=1$, where $\boldsymbol{e}$ is a column vector of ones with proper dimension. We write

$$
\xi_{i, 0}=\frac{\lambda^{i}}{i ! \mu_{1}^{i}}, \quad i=0,1, \ldots, n
$$

and

$$
\begin{gathered}
\xi_{n, j}^{(\boldsymbol{d})}=\frac{\lambda^{n}}{n ! \mu_{1}^{n}} \frac{\lambda^{j}}{\prod_{i=1}^{j} \nu\left(d_{n, i}\right)}, \quad j=1,2, \ldots, m, \\
b^{(\boldsymbol{d})}=\sum_{i=0}^{n} \xi_{i, 0}+\sum_{j=1}^{m} \xi_{n, j}^{(\boldsymbol{d})} .
\end{gathered}
$$

It follows from Subsection 1.1.4 of Chapter 1 in Li [29] that

$$
\begin{aligned}
\pi^{(\boldsymbol{d})}(i, 0) & =\frac{1}{b^{(\boldsymbol{d})}} \xi_{i, 0}, \quad i=0,1, \ldots, n \\
\pi^{(\boldsymbol{d})}(n, j) & =\frac{1}{b^{(\boldsymbol{d})}} \xi_{n, j}^{(\boldsymbol{d})}, \quad j=1,2, \ldots, m .
\end{aligned}
$$

For any two vectors $\boldsymbol{a}=\left(a_{1}, a_{2}, \ldots, a_{K}\right)$ and $\boldsymbol{b}=\left(b_{1}, b_{2}, \ldots, b_{K}\right)$, we say that $\boldsymbol{a} \geq \boldsymbol{b}$ if $a_{i} \geq b_{i}$ for any $1 \leq i \leq K$. The following proposition provides an interesting observation on how a policy $\boldsymbol{d} \in \mathcal{D}$ influences the stationary probability vector $\boldsymbol{\pi}^{(\boldsymbol{d})}$.

Proposition 1 For any two given policies $\boldsymbol{d}_{1}, \boldsymbol{d}_{2} \in \mathcal{D}$ with $\boldsymbol{d}_{1} \geq \boldsymbol{d}_{2}$, then

$$
\pi^{\left(\boldsymbol{d}_{1}\right)}(i, 0) \geq \pi^{\left(\boldsymbol{d}_{2}\right)}(i, 0), \quad i=0,1, \ldots, n .
$$

Proof: For any two given policies $\boldsymbol{d}_{1}, \boldsymbol{d}_{2} \in \mathcal{D}$ with $\boldsymbol{d}_{1} \geq \boldsymbol{d}_{2}$, then it follows from (5) that

$$
\xi_{i, 0}^{\left(\boldsymbol{d}_{1}\right)}=\xi_{i, 0}^{\left(\boldsymbol{d}_{2}\right)}=\frac{\lambda^{i}}{i ! \mu_{1}^{i}}, \quad i=0,1, \ldots, n
$$

If $\boldsymbol{d}_{1} \geq \boldsymbol{d}_{2}$, then for each $j=1,2, \ldots, m$, it is clear that $d_{n, j}^{1} \geq d_{n, j}^{2}$, this gives

$$
\nu\left(d_{n, j}^{1}\right)=n \mu_{1}+\left(d_{n, j}^{1} \wedge j\right) \mu_{2} \geq n \mu_{1}+\left(d_{n, j}^{2} \wedge j\right) \mu_{2}=\nu\left(d_{n, j}^{2}\right)
$$

hence it follows from (6) that

$$
\xi_{n, j}^{\left(\boldsymbol{d}_{1}\right)}=\frac{\lambda^{n}}{n ! \mu_{1}^{n}} \frac{\lambda^{j}}{\prod_{i=1}^{j} \nu\left(d_{n, i}^{1}\right)} \leq \frac{\lambda^{n}}{n ! \mu_{1}^{n}} \frac{\lambda^{j}}{\prod_{i=1}^{j} \nu\left(d_{n, i}^{2}\right)}=\xi_{n, j}^{\left(\boldsymbol{d}_{2}\right)} .
$$


It is easy to see from (7) that

$$
b^{\left(\boldsymbol{d}_{1}\right)}=\sum_{i=0}^{n} \xi_{i, 0}+\sum_{j=1}^{m} \xi_{n, j}^{\left(\boldsymbol{d}_{1}\right)} \leq \sum_{i=0}^{n} \xi_{i, 0}+\sum_{j=1}^{m} \xi_{n, j}^{\left(\boldsymbol{d}_{2}\right)}=b^{\left(\boldsymbol{d}_{2}\right)} .
$$

Thus, it follows (8) that for each $i=0,1, \ldots, n$,

$$
\pi^{\left(\boldsymbol{d}_{1}\right)}(i, 0)=\frac{1}{b^{\left(\boldsymbol{d}_{1}\right)}} \xi_{i, 0} \geq \frac{1}{b^{\left(\boldsymbol{d}_{2}\right)}} \xi_{i, 0}=\pi^{\left(\boldsymbol{d}_{2}\right)}(i, 0) .
$$

This completes the proof.

The following theorem provides a useful observation that some special policies $\boldsymbol{d} \in \mathcal{D}$ have no effect on both the infinitesimal generator $\mathbf{B}^{(\boldsymbol{d})}$ and the stationary probability vector $\boldsymbol{\pi}^{(\boldsymbol{d})}$. Note that this theorem will be necessary and useful for the analysis of policy monotonicity and optimality in our later study, for example, the proof of Theorem 3.

Theorem 1 Suppose that two policies $\boldsymbol{d}_{1}, \boldsymbol{d}_{2} \in \mathcal{D}$ satisfy the following two conditions: For each $j=1,2, \ldots, m$, (a) if $d_{n, j}^{1} \in\{1,2, \ldots, j-1\}$, then we take $d_{n, j}^{2}=d_{n, j}^{1}$; and (b) if $d_{n, j}^{1} \in\{j, j+1, \ldots, m\}$, we take $d_{n, j}^{2}$ as any element of the set $\{j, j+1, \ldots, m\}$. We have

$$
\mathbf{B}^{\left(d_{1}\right)}=\mathbf{B}^{\left(d_{2}\right)}, \quad \boldsymbol{\pi}^{\left(d_{1}\right)}=\boldsymbol{\pi}^{\left(d_{2}\right)} .
$$

Proof: It is easy to see from (3) that the first $n+1$ rows of the matrix $\mathbf{B}^{\left(\boldsymbol{d}_{1}\right)}$ is the same as those of the matrix $\mathbf{B}^{\left(\boldsymbol{d}_{2}\right)}$.

In what follows we compare the latter $m$ rows of the matrix $\mathbf{B}^{\left(\boldsymbol{d}_{1}\right)}$ with those of the matrix $\mathbf{B}^{\left(\boldsymbol{d}_{2}\right)}$. For the two policies $\boldsymbol{d}_{1}, \boldsymbol{d}_{2} \in \mathcal{D}$ satisfying the two conditions (a) and (b), by using $\nu\left(d_{n, j}\right)=n \mu_{1}+\left(d_{n, j} \wedge j\right) \mu_{2}$, it is clear that for $j=1,2, \ldots, m$,

$$
\nu\left(d_{n, j}^{1}\right)=n \mu_{1}+j \mu_{2}=\nu\left(d_{n, j}^{2}\right) .
$$

Thus, it follows from (3) that $\mathbf{B}^{\left(\boldsymbol{d}_{1}\right)}=\mathbf{B}^{\left(\boldsymbol{d}_{2}\right)}$, this also gives that $\boldsymbol{\pi}^{\left(\boldsymbol{d}_{1}\right)}=\boldsymbol{\pi}^{\left(\boldsymbol{d}_{2}\right)}$. This completes the proof.

Based on the problem description in Section 2, we define a suitable reward function for the energy-efficient data center. For convenience of readers, here we explain both energy consumption cost and system operational cost with respect to some key factors of the data center. We summarize five classes of costs in the data center as follows.

(a) Energy consumption cost. It is seen from Section 2 that $P_{1, W}$ and $P_{2, W}$ are the energy consumption rates for the work state in Group 1 and Group 2, respectively; while 
$P_{2, S}$ is the energy consumption rate for the sleep state only in Group 2. In addition, $C_{1}$ is the energy consumption price.

(b) Holding cost. Each job in the data center has to pay a holding $\operatorname{cost} C_{2}^{(1)}\left(\right.$ resp. $\left.C_{2}^{(2)}\right)$ per unit of sojourn time in Group 1 (resp. Group 2). Moreover, we have two assumed conditions: A fast condition $\mu_{1} \geq \mu_{2}$, and a cheap condition $C_{2}^{(1)} \leq C_{2}^{(2)}$.

(c) Transfer cost. If there are some idle servers in Group 1, then the jobs in the servers in Group 2 must be transferred to the idle servers in Group 1 as many as possible. In this case, such each transfer needs to pay a transfer cost $C_{3}$ for each job.

(d) Opportunity cost. Once the data center has contained $n+m$ jobs, then any arriving job has to be lost immediately. This leads to an opportunity cost due to the job loss, hence $C_{4}$ is an opportunity cost for each lost job.

(e) Service price. If a job finishes its service at a server and leaves this system, then the data center gains a fixed service revenue (or earnings) $R$ for each served job, that is, $R$ is the service price.

Based on the above cost and price definition, a reward function with respect to both states and policies is defined as a profit rate (i.e. the total revenues minus the total costs per unit of time). Therefore, the reward function at state $(I(t), J(t))^{(\boldsymbol{d})}$ under policy $\boldsymbol{d}$ is defined as

$$
\begin{aligned}
f^{(\boldsymbol{d})}(i, j)= & R\left[i \mu_{1}+\left(j \wedge d_{i, j}\right) \mu_{2}\right]-\left[n P_{1, W}+d_{i, j} P_{2, W}+\left(m-d_{i, j}\right) P_{2, S}\right] C_{1} \\
& -\left[i C_{2}^{(1)}+j C_{2}^{(2)}\right]-i \mu_{1} 1_{\{j>0\}} C_{3}-\lambda 1_{\{i=n, j=m\}} C_{4}
\end{aligned}
$$

where $1_{\{\cdot\}}$ is an indicator function whose value is 1 when the event in $\{\cdot\}$ happens, otherwise its value is 0 . Furthermore, the job transfer rate from Group 2 to Group 1 is given by $i \mu_{1} 1_{\{j>0\}}$. If $0 \leq i \leq n-1$, then $j=0$ and $i \mu_{1} 1_{\{j>0\}} C_{3}=0$. If $i=n$ and $j=0$, then $i \mu_{1} 1_{\{j>0\}} C_{3}=0$. If $i=n$ and $1 \leq j \leq m$, then $i \mu_{1} 1_{\{j>0\}} C_{3}=n \mu_{1} C_{3}$.

For convenience of readers, it is necessary to explain the reward function from four different cases as follows.

Case (a): For $i=0$ and $j=0$,

$$
f(0,0)=-\left(n P_{1, W}+m P_{2, S}\right) C_{1} .
$$

Case (b): For $i=1,2, \ldots, n$ and $j=0$,

$$
f(i, 0)=R i \mu_{1}-\left(n P_{1, W}+m P_{2, S}\right) C_{1}-i C_{2}^{(1)} .
$$


Note that in Cases (a) and (b), there is no job in Group 2, thus each server of Group 2 is at the sleep state. However, in the following two cases (c) and (d), there are some jobs in Group 2, hence the policy $\boldsymbol{d}$ will play a key role in opening or closing some servers of Group 2 in order to effectively save energy.

Case (c): For $i=n, j=1,2, \ldots, m-1$; and $d_{n, j}=0,1, \ldots, m$,

$$
\begin{aligned}
f^{(\boldsymbol{d})}(n, j)= & R\left[n \mu_{1}+\left(j \wedge d_{n, j}\right) \mu_{2}\right]-\left[n P_{1, W}+d_{n, j} P_{2, W}+\left(m-d_{n, j}\right) P_{2, S}\right] C_{1} \\
& -\left[n C_{2}^{(1)}+j C_{2}^{(2)}\right]-n \mu_{1} C_{3} .
\end{aligned}
$$

To further simplify or compute (13), we need to especially deal with $j \wedge d_{n, j}$. To this end, if $d_{n, j}=1,2, \ldots, j$, then $j \wedge d_{n, j}=d_{n, j}$, hence we have

$$
\begin{aligned}
f^{(\boldsymbol{d})}(n, j)= & R n \mu_{1}+\left[R \mu_{2}-\left(P_{2, W}-P_{2, S}\right) C_{1}\right] d_{n, j} \\
& -\left(n P_{1, W}+m P_{2, S}\right) C_{1}-\left[n C_{2}^{(1)}+j C_{2}^{(2)}\right]-n \mu_{1} C_{3} ;
\end{aligned}
$$

while if $d_{n, j}=j+1, j+2, \ldots, m$, then $j \wedge d_{n, j}=j$, hence we have

$$
\begin{aligned}
f^{(\boldsymbol{d})}(n, j)= & R\left(n \mu_{1}+j \mu_{2}\right)-\left(P_{2, W}-P_{2, S}\right) C_{1} d_{n, j} \\
& -\left(n P_{1, W}+m P_{2, S}\right) C_{1}-\left[n C_{2}^{(1)}+j C_{2}^{(2)}\right]-n \mu_{1} C_{3} .
\end{aligned}
$$

Case (d): For $i=n$ and $j=m$; and $d_{n, m}=0,1, \ldots, m$, we obtain that $m \wedge d_{n, m}=$ $d_{n, m}$, and we have

$$
\begin{aligned}
f^{(\boldsymbol{d})}(n, m)= & R\left(n \mu_{1}+d_{n, m} \mu_{2}\right)-\left[n P_{1, W}+d_{n, m} P_{2, W}+\left(m-d_{n, m}\right) P_{2, S}\right] C_{1} \\
& -\left[n C_{2}^{(1)}+m C_{2}^{(2)}\right]-n \mu_{1} C_{3}-\lambda C_{4} \\
= & R n \mu_{1}+\left[R \mu_{2}-\left(P_{2, W}-P_{2, S}\right) C_{1}\right] d_{n, m} \\
& -\left(n P_{1, W}+m P_{2, S}\right) C_{1}-\left[n C_{2}^{(1)}+m C_{2}^{(2)}\right]-n \mu_{1} C_{3}-\lambda C_{4} .
\end{aligned}
$$

We define an $(n+m+1)$-dimensional column vector composed of the elements $f(i, j)$ and $f^{(\boldsymbol{d})}(i, j)$ as

$$
\boldsymbol{f}^{(\boldsymbol{d})}=\left(f(0,0), f(1,0), \ldots, f(n, 0), f^{(\boldsymbol{d})}(n, 1), \ldots, f^{(\boldsymbol{d})}(n, m)\right)^{T},
$$

where $a^{T}$ denotes transpose of vector or matrix $a$.

In the remainder of this section, the long-run average profit of the data center (or the policy-based continuous-time birth-death process $\left.\left\{\mathbf{X}^{(\boldsymbol{d})}(t): t \geq 0\right\}\right)$ under an energy- 
efficient policy $\boldsymbol{d}$ is defined as

$$
\begin{aligned}
\eta^{\boldsymbol{d}} & =\lim _{T \rightarrow+\infty} E\left\{\frac{1}{T} \int_{0}^{T} f^{(\boldsymbol{d})}\left((I(t), J(t))^{(\boldsymbol{d})}\right) \mathrm{d} t\right\} \\
& =\lim _{T \rightarrow+\infty} E\left\{\frac{1}{T} \int_{0}^{T} f^{(\boldsymbol{d})}\left(\mathbf{X}^{(\boldsymbol{d})}(t)\right) \mathrm{d} t\right\} \\
& =\boldsymbol{\pi}^{(\boldsymbol{d})} \boldsymbol{f}^{(\boldsymbol{d})},
\end{aligned}
$$

where $\boldsymbol{\pi}^{(\boldsymbol{d})}$ and $\boldsymbol{f}^{(\boldsymbol{d})}$ are given by (4) and (17), respectively.

We observe that as the number of working servers in Group 2 decreases, the total revenues and the total costs in the data center will decrease synchronously, vice versa. Thus, there is a tradeoff between the total revenues and the total costs. This motivates us to study an optimal mechanism design for the energy-efficient data center. The objective is to find an optimal energy-efficient policy $\boldsymbol{d}^{*}$ such that the long-run average profit $\eta^{\boldsymbol{d}}$ is maximize, that is,

$$
\boldsymbol{d}^{*}=\underset{\boldsymbol{d} \in \mathcal{D}}{\arg \max }\left\{\eta^{\boldsymbol{d}}\right\}
$$

In fact, it is difficult and challenging to analyze the properties of the optimal energyefficient policy $\boldsymbol{d}^{*}$, and to provide an effective algorithm for computing the optimal policy $\boldsymbol{d}^{*}$. In the next section, we will introduce the sensitivity-based optimization theory to study this energy-efficient optimization problem.

\section{The Poisson Equation and Its Explicit Solution}

In this section, for the energy-efficient data center, we set up a Poisson equation which is derived by means of the law of total probability focusing on some stop times. It is worth noting that the Poisson equation provides a useful relation between the sensitivitybased optimization and the Markov decision processes (MDPs). Also, we use the RGfactorization, given in $\mathrm{Li}$ and $\mathrm{Cao}$ [30] or $\mathrm{Li}$ [29], to solve the Poisson equation and provide the explicit expression for its unique solution.

For $\boldsymbol{d} \in \mathcal{D}$, it follows from Chapter 2 in Cao [5] that for the policy-based continuoustime Markov process $\left\{\mathbf{X}^{(\boldsymbol{d})}(t): t \geq 0\right\}$, we define the performance potential as

$$
\begin{aligned}
g^{(\boldsymbol{d})}(i, j) & =E\left\{\int_{0}^{+\infty}\left[f^{(\boldsymbol{d})}\left((I(t), J(t))^{(\boldsymbol{d})}\right)-\eta^{\boldsymbol{d}}\right] \mathrm{d} t \mid(I(0), J(0))^{(\boldsymbol{d})}=(i, j)\right\} \\
& =E\left\{\int_{0}^{+\infty}\left[f^{(\boldsymbol{d})}\left(\mathbf{X}^{(\boldsymbol{d})}(t)\right)-\eta^{\boldsymbol{d}}\right] \mathrm{d} t \mid \mathbf{X}^{(\boldsymbol{d})}(0)=(i, j)\right\}
\end{aligned}
$$


where $\eta^{\boldsymbol{d}}$ is defined in (18). It is seen from Cao [5] that for any policy $\boldsymbol{d} \in \mathcal{D}, g^{(\boldsymbol{d})}(i, j)$ quantifies the contribution of the initial state $(i, j)$ to the long-run average profit of the data center. Here $g^{(\boldsymbol{d})}(i, j)$ is also called the relative value function or the bias in the traditional MDP theory, see, e.g. Puterman [39]. We further define a column vector $\boldsymbol{g}^{(\boldsymbol{d})}$ with elements $g^{(\boldsymbol{d})}(i, j)$ for $(i, j) \in \boldsymbol{\Omega}$ as

$$
\boldsymbol{g}^{(\boldsymbol{d})}=\left(g^{(\boldsymbol{d})}(0,0), g^{(\boldsymbol{d})}(1,0), \ldots, g^{(\boldsymbol{d})}(n, 0), g^{(\boldsymbol{d})}(n, 1), \ldots, g^{(\boldsymbol{d})}(n, m)\right)^{T} .
$$

We define the first departure time from state $(i, j)$ as

$$
\tau=\inf \left\{t \geq 0:(I(t), J(t))^{(\boldsymbol{d})} \neq(i, j)\right\},
$$

where $(I(0), J(0))^{(\boldsymbol{d})}=(i, j)$. Clearly, $\tau$ is a stop time of the Markov process $\left\{\mathbf{X}^{(\boldsymbol{d})}(t): t \geq 0\right\}$. Based on this, if $(i, j)=(0,0)$, then it is seen from (3) that state $(0,0)$ is the upper boundary state of the birth-death process $\mathbf{B}^{(\boldsymbol{d})}$, hence $(I(\tau), J(\tau))^{(\boldsymbol{d})}=(1,0)$. Similarly, we get a basic relation for the stop time $\tau$ as follows.

$$
(I(\tau), J(\tau))^{(\boldsymbol{d})}= \begin{cases}(1,0), & (i, j)=(0,0), \\ (i-1,0),(i+1,0), & (i, j)=(i, 0), i=1,2, \ldots, n-1, \\ (n-1,0),(n, 1), & (i, j)=(n, 0), \\ (n, j-1),(n, j+1), & (i, j)=(n, j), j=1,2, \ldots, m-1, \\ (n, m-1), & (i, j)=(n, m) .\end{cases}
$$

Now, we derive a Poisson equation to compute the column vector $\boldsymbol{g}^{(\boldsymbol{d})}$ in terms of the stop time $\tau$ and the basic relation (22). By a similar computation to that in Li and Cao [30] or Xia et al. [50], our analysis is decomposed into five parts as follows. 
For $i=1,2, \ldots, n-1$ and $j=0$, we have

$$
\begin{aligned}
g^{(\boldsymbol{d})}(i, 0)= & E\left\{\int_{0}^{+\infty}\left[f^{(\boldsymbol{d})}\left(\mathbf{X}^{(\boldsymbol{d})}(t)\right)-\eta^{\boldsymbol{d}}\right] \mathrm{d} t \mid(I(0), J(0))^{(\boldsymbol{d})}=(i, 0)\right\} \\
= & E\{\tau \mid I(t)=i, J(t)=0\}\left[f(i, 0)-\eta^{\boldsymbol{d}}\right] \\
& +E\left\{\int_{\tau}^{+\infty}\left[f^{(\boldsymbol{d})}\left(\mathbf{X}^{(\boldsymbol{d})}(t)\right)-\eta^{\boldsymbol{d}}\right] \mathrm{d} t \mid(I(\tau), J(\tau))^{(\boldsymbol{d})}\right\} \\
= & \frac{1}{\lambda+i \mu_{1}}\left[f(i, 0)-\eta^{\boldsymbol{d}}\right] \\
& +\frac{\lambda}{\lambda+i \mu_{1}} E\left\{\int_{0}^{+\infty}\left[f^{(\boldsymbol{d})}\left(\mathbf{X}^{(\boldsymbol{d})}(t)\right)-\eta^{\boldsymbol{d}}\right] \mathrm{d} t \mid(I(0), J(0))^{(\boldsymbol{d})}=(i+1,0)\right\} \\
& +\frac{i \mu_{1}}{\lambda+i \mu_{1}} E\left\{\int_{0}^{+\infty}\left[f^{(\boldsymbol{d})}\left(\mathbf{X}^{(\boldsymbol{d})}(t)\right)-\eta^{\boldsymbol{d}}\right] \mathrm{d} t \mid(I(0), J(0))^{(\boldsymbol{d})}=(i-1,0)\right\} \\
= & \frac{1}{\lambda+i \mu_{1}}\left[f(i, 0)-\eta^{\boldsymbol{d}}\right]+\frac{\lambda}{\lambda+i \mu_{1}} g^{(\boldsymbol{d})}(i+1,0)+\frac{i \mu_{1}}{\lambda+i \mu_{1}} g^{(\boldsymbol{d})}(i-1,0), \quad(23)
\end{aligned}
$$

where for the birth-death process $\left\{\mathbf{X}^{(\boldsymbol{d})}(t): t \geq 0\right\}$, it is easy to see that

$$
\begin{gathered}
\int_{0}^{\tau}\left[f^{(\boldsymbol{d})}\left((I(t), J(t))^{(\boldsymbol{d})}\right)-\eta^{\boldsymbol{d}}\right] \mathrm{d} t=\tau\left[f(i, 0)-\eta^{\boldsymbol{d}}\right], \\
E\{\tau \mid I(t)=i, J(t)=0\}=\frac{1}{\lambda+i \mu_{1}} .
\end{gathered}
$$

Thus, we obtain

$$
i \mu_{1} g^{(\boldsymbol{d})}(i-1,0)-\left(\lambda+i \mu_{1}\right) g^{(\boldsymbol{d})}(i, 0)+\lambda g^{(\boldsymbol{d})}(i+1,0)=\eta^{\boldsymbol{d}}-f(i, 0) .
$$

Base on (23), with a boundary consideration, for $i=0$ and $j=0$, we have

$$
-\lambda g^{(\boldsymbol{d})}(0,0)+\lambda g^{(\boldsymbol{d})}(1,0)=\eta^{\boldsymbol{d}}-f(0,0) .
$$

For $i=n$ and $j=0$, we have

$$
n \mu_{1} g^{(\boldsymbol{d})}(n-1,0)-\left(\lambda+n \mu_{1}\right) g^{(\boldsymbol{d})}(n, 0)+\lambda g^{(\boldsymbol{d})}(n, 1)=\eta^{\boldsymbol{d}}-f(n, 0) .
$$


For $i=n$ and $j=1,2, \ldots, m-1$, we have

$$
\begin{aligned}
g^{(\boldsymbol{d})}(n, j)= & E\left\{\int_{0}^{+\infty}\left[f^{(\boldsymbol{d})}\left(\mathbf{X}^{(\boldsymbol{d})}(t)\right)-\eta^{\boldsymbol{d}}\right] \mathrm{d} t \mid(I(0), J(0))^{(\boldsymbol{d})}=(n, j)\right\} \\
= & E\{\tau \mid I(t)=n, J(t)=j\}\left[f^{(\boldsymbol{d})}(n, j)-\eta^{\boldsymbol{d}}\right] \\
& +E\left\{\int_{\tau}^{+\infty}\left[f^{(\boldsymbol{d})}\left(\mathbf{X}^{(\boldsymbol{d})}(t)\right)-\eta^{\boldsymbol{d}}\right] \mathrm{d} t \mid(I(\tau), J(\tau))^{(\boldsymbol{d})}\right\} \\
= & \frac{1}{\lambda+\nu\left(d_{n, j}\right)}\left[f^{(\boldsymbol{d})}(n, j)-\eta^{\boldsymbol{d}}\right] \\
& +\frac{\lambda}{\lambda+\nu\left(d_{n, j}\right)} E\left\{\int_{0}^{+\infty}\left[f^{(\boldsymbol{d})}\left(\mathbf{X}^{(\boldsymbol{d})}(t)\right)-\eta^{\boldsymbol{d}}\right] \mathrm{d} t \mid(I(0), J(0))^{(\boldsymbol{d})}=(n, j+1)\right\} \\
& +\frac{\nu\left(d_{n, j}\right)}{\lambda+\nu\left(d_{n, j}\right)} E\left\{\int_{0}^{+\infty}\left[f^{(\boldsymbol{d})}\left(\mathbf{X}^{(\boldsymbol{d})}(t)\right)-\eta^{\boldsymbol{d}}\right] \mathrm{d} t \mid(I(0), J(0))^{(\boldsymbol{d})}=(n, j-1)\right\} \\
= & \frac{1}{\lambda+\nu\left(d_{n, j}\right)}\left[f^{(\boldsymbol{d})}(n, j)-\eta^{\boldsymbol{d}}\right]+\frac{\lambda}{\lambda+\nu\left(d_{n, j}\right)} g^{(\boldsymbol{d})}(n, j+1) \\
& +\frac{\nu\left(d_{n, j}\right)}{\lambda+\nu\left(d_{n, j}\right)} g^{(\boldsymbol{d})}(n, j-1),
\end{aligned}
$$

where

$$
E\{\tau \mid I(t)=n, J(t)=j\}=\frac{1}{\lambda+\nu\left(d_{n, j}\right)} .
$$

It follows from (27) that

$$
\nu\left(d_{n, j}\right) g^{(\boldsymbol{d})}(n, j-1)-\left[\lambda+\nu\left(d_{n, j}\right)\right] g^{(\boldsymbol{d})}(n, j)+\lambda g^{(\boldsymbol{d})}(n, j+1)=\eta^{\boldsymbol{d}}-f^{(\boldsymbol{d})}(n, j)
$$

For $i=n$ and $j=m$, with a boundary consideration, a similar analysis to (29) gives

$$
\nu\left(d_{n, m}\right) g^{(\boldsymbol{d})}(n, m-1)-\nu\left(d_{n, m}\right) g^{(\boldsymbol{d})}(n, m)=\eta^{\boldsymbol{d}}-f^{(\boldsymbol{d})}(n, m) .
$$

Note that $\nu\left(d_{n, m}\right)=n \mu_{1}+\left(d_{n, m} \wedge m\right) \mu_{2}=n \mu_{1}+d_{n, m} \mu_{2}$ due to the fact that $d_{n, m} \in$ $\{0,1, \ldots, m\}$.

It follows from (24), (25), (26), (29) and (30) that

$$
\mathbf{B}^{(d)} \boldsymbol{g}^{(\boldsymbol{d})}=\eta^{\boldsymbol{d}} \boldsymbol{e}-\boldsymbol{f}^{(\boldsymbol{d})}
$$

or

$$
-\mathbf{B}^{(d)} g^{(d)}=f^{(d)}-\eta^{d} e
$$

where $\boldsymbol{f}^{(\boldsymbol{d})}$ is given in (17) and $\mathbf{B}^{(\boldsymbol{d})}$ is given in (3).

To solve the system of linear equations (31), we note that $\operatorname{rank}\left(\mathbf{B}^{(\boldsymbol{d})}\right)=n+m$ and $\operatorname{det}\left(\mathbf{B}^{(\boldsymbol{d})}\right)=0$ due to that the size of the matrix $\mathbf{B}^{(\boldsymbol{d})}$ is $n+m+1$. Hence, this system of 
linear equations (31) exists infinitely-many solutions with a free constant of an additive term. Let $\mathcal{B}$ be a matrix obtained through omitting the first row and the first column vectors of the matrix $\mathbf{B}^{(\boldsymbol{d})}$. Then,

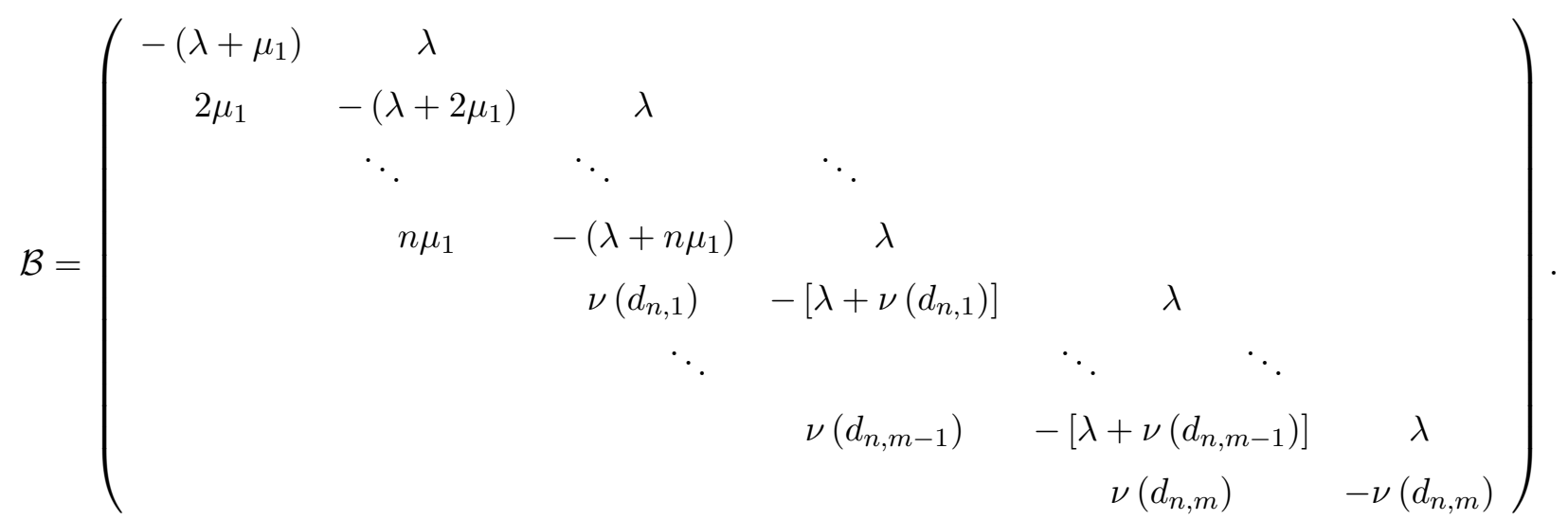

Obviously, $\operatorname{rank}(\mathcal{B})=n+m$ and the size of the matrix $\mathcal{B}$ is $n+m$. Hence, the matrix $\mathcal{B}$ is invertible.

Let $\boldsymbol{h}^{(\boldsymbol{d})}$ and $\boldsymbol{\varphi}^{(\boldsymbol{d})}$ be two column vectors of size $n+m$ obtained through omitting the first element of the two column vectors $\boldsymbol{f}^{(\boldsymbol{d})}-\eta^{\boldsymbol{d}} \boldsymbol{e}$ and $\boldsymbol{g}^{(\boldsymbol{d})}$ with size $n+m+1$, respectively. Then,

$$
\boldsymbol{h}^{(\boldsymbol{d})}=\left(\begin{array}{c}
f(1,0)-\eta^{\boldsymbol{d}} \\
\vdots \\
f(n, 0)-\eta^{\boldsymbol{d}} \\
f^{(\boldsymbol{d})}(n, 1)-\eta^{\boldsymbol{d}} \\
\vdots \\
f^{(\boldsymbol{d})}(n, m)-\eta^{\boldsymbol{d}}
\end{array}\right) \stackrel{\text { def }}{=}\left(\begin{array}{c}
h_{1}^{(\boldsymbol{d})} \\
\vdots \\
h_{n}^{(\boldsymbol{d})} \\
h_{n+1}^{(\boldsymbol{d})} \\
\vdots \\
h_{n+m}^{(\boldsymbol{d})}
\end{array}\right), \quad \boldsymbol{\varphi}^{(\boldsymbol{d})}=\left(\begin{array}{c}
g^{(\boldsymbol{d})}(1,0) \\
\vdots \\
g^{(\boldsymbol{d})}(n, 0) \\
g^{(\boldsymbol{d})}(n, 1) \\
\vdots \\
g^{(\boldsymbol{d})}(n, m)
\end{array}\right) .
$$

Therefore, it follows from (31) that

$$
-\mathcal{B} \varphi^{(\boldsymbol{d})}=\boldsymbol{h}^{(\boldsymbol{d})}+\mu_{1} \boldsymbol{e}_{1} g^{(\boldsymbol{d})}(0,0),
$$

where $\boldsymbol{e}_{1}$ is a column vector with the first element be one and all the others be zero. Note that the matrix $-\mathcal{B}$ is invertible and $(-\mathcal{B})^{-1}>0$, thus, the system of linear equations (32) always exists one unique solution

$$
\varphi^{(\boldsymbol{d})}=(-\mathcal{B})^{-1} \boldsymbol{h}^{(\boldsymbol{d})}+\mu_{1}(-\mathcal{B})^{-1} \boldsymbol{e}_{1} \cdot \Im
$$

where $g^{(\boldsymbol{d})}(0,0)=\Im$ is any given constant. For convenience of computation, we take $g^{(\boldsymbol{d})}(0,0)=\Im=1$. In this case, we have

$$
\varphi^{(\boldsymbol{d})}=(-\mathcal{B})^{-1} \boldsymbol{h}^{(\boldsymbol{d})}+\mu_{1}(-\mathcal{B})^{-1} \boldsymbol{e}_{1} .
$$


To provide an explicit expression for the unique solution to the system of linear equations (32), it is easy to see from (34) that we need to first establish an explicit expression for the invertible matrix $(-\mathcal{B})^{-1}$. While the explicit expression of the invertible matrix $(-\mathcal{B})^{-1}$ can be obtained by means of the RG-factorization, which is given in Li and Cao 30] for QBD processes, and more generally, Li [29] for general Markov processes.

To express the invertible matrix $(-\mathcal{B})^{-1}$, we write the UL-type $U$-measure as

$$
\begin{aligned}
& U_{n+m}=-\nu\left(d_{n, m}\right) \\
& U_{n+m-1}=-\left[\lambda+\nu\left(d_{n, m-1}\right)\right]+\lambda\left(-U_{n+m}\right)^{-1} \nu\left(d_{n, m}\right) \\
& U_{k}=A_{1}^{(k)}+\lambda\left(-U_{k+1}\right)^{-1} A_{2}^{(k+1)}, \quad k=n+m-2, n+m-3, \ldots, 1,
\end{aligned}
$$

and the UL-type $R$ - and $G$-measures as

$$
\begin{aligned}
& R_{k}=\lambda\left(-U_{k+1}\right)^{-1}, \quad k=1,2, \ldots, n+m-1, \\
& G_{k}=\left(-U_{k}\right)^{-1} A_{2}^{(k)}, \quad k=2,3, \ldots, n+m,
\end{aligned}
$$

where

$$
\begin{aligned}
& A_{1}^{(k)}= \begin{cases}-\left(\lambda+k \mu_{1}\right), & k=1,2, \ldots, n, \\
-\left[\lambda+\nu\left(d_{n, k-n}\right)\right], & k=n+1, n+2, \ldots, n+m-1,\end{cases} \\
& A_{2}^{(k)}= \begin{cases}k \mu_{1}, & k=2,3, \ldots, n, \\
\nu\left(d_{n, k-n}\right), & k=n+1, n+2, \ldots, n+m .\end{cases}
\end{aligned}
$$

Thus, the UL-type RG-factorization of the birth-death process $\mathcal{B}$ is given by

$$
\mathcal{B}=\left(I-R_{U}\right) U_{D}\left(I-G_{L}\right),
$$

where

$$
R_{U}=\left(\begin{array}{ccccc}
0 & R_{1} & & & \\
& 0 & R_{2} & & \\
& & \ddots & \ddots & \\
& & & 0 & R_{n+m-1} \\
& & & & 0
\end{array}\right), G_{L}=\left(\begin{array}{ccccc}
0 & & & & \\
G_{2} & 0 & & & \\
& G_{3} & 0 & & \\
& & \ddots & \ddots & \\
& & & G_{n+m} & 0
\end{array}\right)
$$

and

$$
U_{D}=\operatorname{diag}\left(U_{1}, U_{2}, \ldots, U_{n+m}\right) .
$$

Therefore, we obtain

$$
(-\mathcal{B})^{-1}=\left(I-G_{L}\right)^{-1}\left(-U_{D}\right)^{-1}\left(I-R_{U}\right)^{-1} .
$$


Let

$$
\begin{aligned}
& X_{k}^{(l)}=R_{l} R_{l+1} \cdots R_{l+k-2}, 1 \leq l \leq n+m-1,2 \leq k \leq n+m, \\
& Y_{k}^{(l)}=G_{l} G_{l-1} \cdots G_{l-k+2}, 2 \leq k \leq l \leq n+m .
\end{aligned}
$$

Then,

$$
\begin{aligned}
\left(I-G_{L}\right)^{-1} & =\left(\begin{array}{ccccc}
1 & & & & \\
Y_{2}^{(2)} & 1 & & & \\
Y_{3}^{(3)} & Y_{2}^{(3)} & 1 & & \\
\vdots & \vdots & \ddots & \ddots & \\
Y_{n+m}^{(n+m)} & Y_{n+m-1}^{(n+m)} & \cdots & Y_{2}^{(n+m)} & 1
\end{array}\right), \\
\left(I-R_{U}\right)^{-1} & =\left(\begin{array}{cccccc}
1 & X_{2}^{(1)} & X_{3}^{(1)} & \cdots & X_{n+m}^{(1)} \\
& 1 & X_{2}^{(2)} & \cdots & X_{n+m-1}^{(2)} \\
& & 1 & \cdots & X_{n+m-2}^{(3)} \\
& & & \ddots & \vdots \\
& & & & 1
\end{array}\right),
\end{aligned}
$$

and

$$
\left(-U_{D}\right)^{-1}=\operatorname{diag}\left(\left(-U_{1}\right)^{-1},\left(-U_{2}\right)^{-1}, \ldots,\left(-U_{n+m}\right)^{-1}\right) .
$$

Thus, we obtain the explicit expression

$$
\begin{aligned}
& (-\mathcal{B})^{-1}=\left(\begin{array}{ccccc}
1 & & & & \\
Y_{2}^{(2)} & 1 & & & \\
Y_{3}^{(3)} & Y_{2}^{(3)} & 1 & & \\
\vdots & \vdots & \ddots & \ddots & \\
Y_{n+m}^{(n+m)} & Y_{n+m-1}^{(n+m)} & \cdots & Y_{2}^{(n+m)} & 1
\end{array}\right) \operatorname{diag}\left(\left(-U_{1}\right)^{-1},\left(-U_{2}\right)^{-1}, \ldots,\left(-U_{n+m}\right)^{-1}\right) \\
& \left(\begin{array}{ccccc}
1 & X_{2}^{(1)} & X_{3}^{(1)} & \cdots & X_{n+m}^{(1)} \\
& 1 & X_{2}^{(2)} & \cdots & X_{n+m-1}^{(2)} \\
& & 1 & \cdots & X_{n+m-2}^{(3)} \\
& & & \ddots & \vdots \\
& & & & 1
\end{array}\right)
\end{aligned}
$$

It is worthwhile to note that for the $U-, R$ - and $G$-measures discussed above, each of them depends on the policy $\boldsymbol{d} \in \mathcal{D}$. For simplification of descriptions, we omit such superscript $\boldsymbol{d}$ in their formulae or equations. 
Remark 3 In general, it is difficult to solve the high-dimensional system of linear equations (32) for a general Markov process. However, the RG-factorization method is usually very effective for solving such problem for a level-dependent QBD process, in which the expression of the invertible matrix $(-\mathcal{B})^{-1}$ is similar to that in (35) with elements changed from scalar to block matrices. In addition, it is also a key that the vector $\varphi^{(\boldsymbol{d})}$ can be numerically computed from (33) and (35) by using the RG-factorization. See Li and Cao [30] for more details.

The following theorem provides an explicit expression for the vector $\varphi^{(d)}$ under a constraint condition $g^{(\boldsymbol{d})}(0,0)=1$. Note that this expression is very useful for applications of the sensitivity-based optimization theory in our later study.

Theorem 2 If $g^{(\boldsymbol{d})}(0,0)=1$, then for $k=1$,

$$
g^{(\boldsymbol{d})}(1,0)=\left(-U_{1}\right)^{-1}\left[h_{1}^{(\boldsymbol{d})}+\sum_{j=2}^{m+n} X_{j}^{(1)} h_{j}^{(\boldsymbol{d})}\right]+G_{1}
$$

For $2 \leq k \leq n$,

$$
\begin{aligned}
g^{(\boldsymbol{d})}(k, 0)= & \left(-U_{k}\right)^{-1}\left[h_{k}^{(\boldsymbol{d})}+\sum_{j=2}^{m+n-k+1} X_{j}^{(k)} h_{j+k-1}^{(\boldsymbol{d})}\right] \\
& +\sum_{i=1}^{k-1} Y_{k-i+1}^{(k)}\left(-U_{i}\right)^{-1}\left[h_{i}^{(\boldsymbol{d})}+\sum_{j=2}^{m+n-i+1} X_{j}^{(i)} h_{j+i-1}^{(\boldsymbol{d})}\right]+\prod_{j=1}^{k} G_{j}
\end{aligned}
$$

For $n+1 \leq k \leq n+m-1$,

$$
\begin{aligned}
g^{(\boldsymbol{d})}(n, k-n)= & \left(-U_{k}\right)^{-1}\left[h_{k}^{(\boldsymbol{d})}+\sum_{j=2}^{m+n-k+1} X_{j}^{(k)} h_{j+k-1}^{(\boldsymbol{d})}\right] \\
& +\sum_{i=1}^{k-1} Y_{k-i+1}^{(k)}\left(-U_{i}\right)^{-1}\left[h_{i}^{(\boldsymbol{d})}+\sum_{j=2}^{m+n-i+1} X_{j}^{(i)} h_{j+i-1}^{(\boldsymbol{d})}\right]+\prod_{j=1}^{k} G_{j} ;
\end{aligned}
$$

For $k=n+m$

$$
\begin{aligned}
g^{(\boldsymbol{d})}(n, m) & =\left(-U_{n+m}\right)^{-1} h_{n+m}^{(\boldsymbol{d})}+\sum_{i=1}^{n+m-1} Y_{n+m-i+1}^{(n+m)}\left(-U_{i}\right)^{-1} \\
& \times\left[h_{i}^{(\boldsymbol{d})}+\sum_{j=2}^{m+n-i+1} X_{j}^{(i)} h_{j+i-1}^{(\boldsymbol{d})}\right]+\prod_{j=1}^{n+m} G_{j} .
\end{aligned}
$$


Proof: It is seen from (34) that we need to compute two parts: $\mu_{1}(-\mathcal{B})^{-1} \boldsymbol{e}_{1}$ and $(-\mathcal{B})^{-1} \boldsymbol{h}^{(\boldsymbol{d})}$. For the first part, we obtain

$$
\mu_{1}(-\mathcal{B})^{-1} \boldsymbol{e}_{1}=\mu_{1}\left(-U_{1}\right)^{-1}\left(1, Y_{2}^{(2)}, Y_{3}^{(3)}, \ldots, Y_{n+m}^{(n+m)}\right)^{T} .
$$

Since $G_{1}=\left(-U_{1}\right)^{-1} \mu_{1}$ and $Y_{k}^{(k)}=\prod_{j=2}^{k} G_{j}$, we obtain

$$
\mu_{1}(-\mathcal{B})^{-1} \boldsymbol{e}_{1}=\left(G_{1}, G_{2} G_{1}, G_{3} G_{2} G_{1}, \ldots, \prod_{j=1}^{m+n} G_{j}\right)^{T}
$$

For the second part, we have

$$
(-\mathcal{B})^{-1} \boldsymbol{h}^{(\boldsymbol{d})}=\left(\begin{array}{c}
\left(-U_{1}\right)^{-1}\left[h_{1}^{(\boldsymbol{d})}+\sum_{j=2}^{m+n} X_{j}^{(1)} h_{j}^{(\boldsymbol{d})}\right] \\
\vdots \\
\left(-U_{k}\right)^{-1}\left[h_{k}^{(\boldsymbol{d})}+\sum_{j=2}^{m+n-k+1} X_{j}^{(k)} h_{j+k-1}^{(\boldsymbol{d})}\right] \\
+\sum_{i=1}^{k-1} Y_{k-i+1}^{(k)}\left(-U_{i}\right)^{-1}\left[h_{i}^{(\boldsymbol{d})}+\sum_{j=2}^{m+n-i+1} X_{j}^{(i)} h_{j+i-1}^{(\boldsymbol{d})}\right] \\
\vdots \\
\left(-U_{n+m}\right)^{-1} h_{n+m}^{(\boldsymbol{d})}+\sum_{i=1}^{n+m-1} Y_{n+m-i+1}^{(n+m)}\left(-U_{i}\right)^{-1} \\
\times\left[h_{i}^{(\boldsymbol{d})}+\sum_{j=2}^{m+n-i+1} X_{j}^{(i)} h_{j+i-1}^{(\boldsymbol{d})}\right]
\end{array}\right) .
$$

Therefore, a simple computation for the vector $\varphi^{(\boldsymbol{d})}=(-\mathcal{B})^{-1} \boldsymbol{h}^{(\boldsymbol{d})}+\mu_{1}(-\mathcal{B})^{-1} \boldsymbol{e}_{1}$ can derive our desired results. This completes the proof.

Remark 4 (1) Theorem 2 provides an effective method of solving the continuous-time Poisson equation $-\mathbf{B}^{(\boldsymbol{d})} \boldsymbol{g}^{(\boldsymbol{d})}=\boldsymbol{f}^{(\boldsymbol{d})}-\eta^{\boldsymbol{d}} \boldsymbol{e}$, through an equation transformation

$$
\left\{\begin{array}{l}
g^{(\boldsymbol{d})}(0,0)=\Im \\
\boldsymbol{\varphi}^{(\boldsymbol{d})}=(-\mathcal{B})^{-1} \boldsymbol{h}^{(\boldsymbol{d})}+\mu_{1}(-\mathcal{B})^{-1} \boldsymbol{e}_{1} \cdot \Im
\end{array}\right.
$$

where $\Im$ is any given constant, and $(-\mathcal{B})^{-1}$ can be effectively computed by means of the $R G$-factorization given in $L i$ [29].

It is necessary to set up a general solution to the system of linear equations (36). Let $\boldsymbol{\varphi}_{0}^{(\boldsymbol{d})}$ be the unique solution to the system of linear equations $-\mathcal{B} \varphi_{0}^{(\boldsymbol{d})}=\boldsymbol{h}^{(\boldsymbol{d})}$. Then, the general solution is given by $\boldsymbol{\varphi}^{(\boldsymbol{d})}=\boldsymbol{\varphi}_{0}^{(\boldsymbol{d})}+\mu_{1}(-\mathcal{B})^{-1} \boldsymbol{e}_{1} \cdot \Im$.

(2) To deal with the Poisson equation, some authors (e.g., see Chapter 2 of Cao [5] and Hunter [22]) provided a fundamental matrix method to give a special solution under a constraint condition $\boldsymbol{\pi}^{(\boldsymbol{d})} \boldsymbol{g}^{(\boldsymbol{d})}=\eta^{\boldsymbol{d}}$, by which the Poisson equation is well related to the 
well-known fundamental matrix (e.g., $(\boldsymbol{I}-\boldsymbol{P}+\boldsymbol{e} \boldsymbol{\pi}) \boldsymbol{g}=\boldsymbol{f}$ or $(-\boldsymbol{Q}+\boldsymbol{e} \boldsymbol{\pi}) \boldsymbol{g}=\boldsymbol{f})$. From the fundamental matrix method, our undetermined constant $\Im$ can be determined by

$$
\Im=\frac{\eta^{\boldsymbol{d}}-\varpi^{(\boldsymbol{d})}(-\mathcal{B})^{-1} \boldsymbol{h}^{(\boldsymbol{d})}}{\pi^{(\boldsymbol{d})}(0,0)+\mu_{1} \varpi^{(\boldsymbol{d})}(-\mathcal{B})^{-1} \boldsymbol{e}_{1}},
$$

where $\varpi^{(\boldsymbol{d})}$ is a row vector obtained through omitting the first element of the stationary probability vector $\boldsymbol{\pi}^{(\boldsymbol{d})}$, that is,

$$
\varpi^{(\boldsymbol{d})}=\left(\pi^{(\boldsymbol{d})}(1,0), \ldots, \pi^{(\boldsymbol{d})}(n, 0), \pi^{(\boldsymbol{d})}(n, 1), \ldots, \pi^{(\boldsymbol{d})}(n, m)\right) .
$$

It is worth noting that in solving the Poisson equation, our RG-factorization method is superior to the fundamental matrix method because the RG-factorization given in Li [29] can easily deal with the inverse of a high-dimensional transition matrix; while computing the inverse $(\boldsymbol{I}-\boldsymbol{P}+\boldsymbol{e} \boldsymbol{\pi})^{-1}$ or $(-\boldsymbol{Q}+\boldsymbol{e} \boldsymbol{\pi})^{-1}$, however, is very difficult for a matrix $\boldsymbol{P}$ or $\boldsymbol{Q}$ with large size, and it also needs to first compute the stationary probability vector $\boldsymbol{\pi}$.

\section{$5 \quad$ Impact of Service Price}

In this section, we define a perturbation realization factor of the policy-based birthdeath process, and analyze how the service price impacts on the perturbation realization factor. Note that the results given in this section will be utilized for establishing the optimal policy of the energy-efficient data center in the later section.

For the performance potential vector $\boldsymbol{\varphi}^{(\boldsymbol{d})}$ under a constraint condition $g^{(\boldsymbol{d})}(0,0)=1$, we define a perturbation realization factor as

$$
G^{(\boldsymbol{d})}(n, j) \stackrel{\text { def }}{=} g^{(\boldsymbol{d})}(n, j-1)-g^{(\boldsymbol{d})}(n, j), j=1,2, \ldots, m .
$$

It follows from Theorem 2 that

$$
\begin{aligned}
g^{(\boldsymbol{d})}(n, j-1)= & \left(-U_{n+j-1}\right)^{-1}\left[h_{n+j-1}^{(\boldsymbol{d})}+\sum_{k=2}^{m-j+2} X_{k}^{(n+j-1)} h_{n+j+k-2}^{(\boldsymbol{d})}\right] \\
& +\sum_{i=1}^{n+j-2} Y_{n+j-i}^{(n+j-1)}\left(-U_{i}\right)^{-1}\left[h_{i}^{(\boldsymbol{d})}+\sum_{k=2}^{m+n-i+1} X_{k}^{(i)} h_{k+i-1}^{(\boldsymbol{d})}\right]+\prod_{k=1}^{n+j-1} G_{k}
\end{aligned}
$$

and

$$
\begin{aligned}
g^{(\boldsymbol{d})}(n, j)= & \left(-U_{n+j}\right)^{-1}\left[h_{n+j}^{(\boldsymbol{d})}+\sum_{k=2}^{m-j+1} X_{k}^{(n+j)} h_{n+j+k-1}^{(\boldsymbol{d})}\right] \\
& +\sum_{i=1}^{n+j-1} Y_{n+j-i+1}^{(n+j)}\left(-U_{i}\right)^{-1}\left[h_{i}^{(\boldsymbol{d})}+\sum_{k=2}^{m+n-i+1} X_{k}^{(i)} h_{k+i-1}^{(\boldsymbol{d})}\right]+\prod_{k=1}^{n+j} G_{k} .
\end{aligned}
$$


To express the perturbation realization factor $G^{(\boldsymbol{d})}(n, j)$ by means of the service price $R$, we write

$$
A_{0,0}=0, \quad B_{0,0}=\left(n P_{1, W}+m P_{2, S}\right) C_{1}>0
$$

For $i=1,2, \ldots, n$,

$$
A_{i, 0}=i \mu_{1}>0, \quad B_{i, 0}=\left(n P_{1, W}+m P_{2, S}\right) C_{1}+i C_{2}^{(1)}>0
$$

For $j=1,2, \ldots, m$ and $d_{n, j}=0,1, \ldots, m$,

$$
\begin{gathered}
A_{n, j}^{(\boldsymbol{d})}=n \mu_{1}+\left(j \wedge d_{n, j}\right) \mu_{2}>0 \\
B_{n, j}^{(\boldsymbol{d})}=\left[n P_{1, W}+d_{n, j} P_{2, W}+\left(m-d_{n, j}\right) P_{2, S}\right] C_{1}+n C_{2}^{(1)}+j C_{2}^{(2)} \\
+n \mu_{1} C_{3}+\lambda 1_{\{i=n, j=m\}} C_{4}>0 .
\end{gathered}
$$

Then, for $i=0,1, \ldots, n$ and $j=0$,

$$
f(i, 0)=R A_{i, 0}-B_{i, 0}
$$

For $j=1,2, \ldots, m$,

$$
f^{(\boldsymbol{d})}(n, j)=R A_{n, j}^{(\boldsymbol{d})}-B_{n, j}^{(\boldsymbol{d})}
$$

Thus, we obtain

$$
\begin{aligned}
\eta^{\boldsymbol{d}} & =\boldsymbol{\pi}^{(\boldsymbol{d})} \boldsymbol{f}^{(\boldsymbol{d})} \\
& =\sum_{i=0}^{n} \pi(i, 0) f(i, 0)+\sum_{j=1}^{m} \pi(n, j) f^{(\boldsymbol{d})}(n, j) \\
& =R D^{(\boldsymbol{d})}-F^{(\boldsymbol{d})},
\end{aligned}
$$

where

$$
D^{(\boldsymbol{d})}=\sum_{i=0}^{n} \pi(i, 0) A_{i, 0}+\sum_{j=1}^{m} \pi(n, j) A_{n, j}^{(\boldsymbol{d})}>0
$$

and

$$
F^{(\boldsymbol{d})}=\sum_{i=0}^{n} \pi(i, 0) B_{i, 0}+\sum_{j=1}^{m} \pi(n, j) B_{n, j}^{(\boldsymbol{d})}>0 .
$$


Then,

$$
\boldsymbol{h}^{(\boldsymbol{d})}=\left(\begin{array}{c}
h_{1}^{(\boldsymbol{d})} \\
\vdots \\
h_{n}^{(\boldsymbol{d})} \\
h_{n+1}^{(\boldsymbol{d})} \\
\vdots \\
h_{n+m}^{(\boldsymbol{d})}
\end{array}\right)=\left(\begin{array}{c}
f(1,0)-\eta^{\boldsymbol{d}} \\
\vdots \\
f(n, 0)-\eta^{\boldsymbol{d}} \\
f^{(\boldsymbol{d})}(n, 1)-\eta^{\boldsymbol{d}} \\
\vdots \\
f^{(\boldsymbol{d})}(n, m)-\eta^{\boldsymbol{d}}
\end{array}\right)=\left(\begin{array}{c}
R\left[A_{1,0}-D^{(\boldsymbol{d})}\right]-\left[B_{1,0}-F^{(\boldsymbol{d})}\right] \\
\vdots \\
R\left[A_{n, 0}-D^{(\boldsymbol{d})}\right]-\left[B_{n, 0}-F^{(\boldsymbol{d})}\right] \\
R\left[A_{n, 1}^{(\boldsymbol{d})}-D^{(\boldsymbol{d})}\right]-\left[B_{n, 1}^{(\boldsymbol{d})}-F^{(\boldsymbol{d})}\right] \\
\vdots \\
R\left[A_{n, m}^{(\boldsymbol{d})}-D^{(\boldsymbol{d})}\right]-\left[B_{n, m}^{(\boldsymbol{d})}-F^{(\boldsymbol{d})}\right]
\end{array}\right) .
$$

If a job finishes its service at a server and leaves this system immediately, then the data center can obtain a fixed revenue $R$ from each job. Obviously, $R$ is the service price provided by the data center. Now, we study the influence of the service price $R$ on the perturbation realization factor $G^{(\boldsymbol{d})}(n, j)$. Note that all the numbers $\left(-U_{k}\right)^{-1}, X_{j}^{(k)}, Y_{j}^{(k)}$ and $G_{j}$ are positive and are independent of the service price $R$, while all the numbers $h_{j}^{(d)}$ are the linear functions of $R$.

We write

$$
\begin{aligned}
W_{n, j}^{(\boldsymbol{d})}= & \left(-U_{n+j}\right)^{-1}\left\{\left[A_{n, j}^{(\boldsymbol{d})}-D^{(\boldsymbol{d})}\right]+\sum_{k=2}^{m-j+1} X_{k}^{(n+j)}\left[A_{n, j+k-1}^{(\boldsymbol{d})}-D^{(\boldsymbol{d})}\right]\right\} \\
& +\sum_{i=1}^{n} Y_{n+j-i+1}^{(n+j)}\left(-U_{i}\right)^{-1}\left[A_{i, 0}-D^{(\boldsymbol{d})}\right]+\sum_{i=n+1}^{n+j-1} Y_{n+j-i+1}^{(n+j)}\left(-U_{i}\right)^{-1}\left[A_{n, i-n}^{(\boldsymbol{d})}-D^{(\boldsymbol{d})}\right] \\
& +\sum_{3 \leq k+i \leq n+1} \sum_{i=1}^{n+j-1} Y_{n+j-i+1}^{(n+j)}\left(-U_{i}\right)^{-1} \sum_{k=2}^{m+n-i+1} X_{k}^{(i)}\left[A_{k+i-1,0}-D^{(\boldsymbol{d})}\right] \\
& +\sum_{n+2 \leq k+i \leq n+m+1} \sum_{i=1}^{n+j-1} Y_{n+j-i+1}^{(n+j)}\left(-U_{i}\right)^{-1} \sum_{k=2}^{m+n-i+1} X_{k}^{(i)}\left[A_{n, k+i-1-n}^{(\boldsymbol{d})}-D^{(\boldsymbol{d})}\right]
\end{aligned}
$$

and

$$
\begin{aligned}
V_{n, j}^{(\boldsymbol{d})}= & \left(-U_{n+j}\right)^{-1}\left\{\left[B_{n, j}^{(\boldsymbol{d})}-F^{(\boldsymbol{d})}\right]+\sum_{k=2}^{m-j+1} X_{k}^{(n+j)}\left[B_{n, j+k-1}^{(\boldsymbol{d})}-F^{(\boldsymbol{d})}\right]\right\} \\
& +\sum_{i=1}^{n} Y_{n+j-i+1}^{(n+j)}\left(-U_{i}\right)^{-1}\left[B_{i, 0}-F^{(\boldsymbol{d})}\right]+\sum_{i=n+1}^{n+j-1} Y_{n+j-i+1}^{(n+j)}\left(-U_{i}\right)^{-1}\left[B_{n, i-n}^{(\boldsymbol{d})}-F^{(\boldsymbol{d})}\right] \\
& +\sum_{3 \leq k+i \leq n+1} \sum_{i=1}^{n+j-1} Y_{n+j-i+1}^{(n+j)}\left(-U_{i}\right)^{-1} \sum_{k=2}^{m+n-i+1} X_{k}^{(i)}\left[B_{k+i-1,0}-F^{(\boldsymbol{d})}\right] \\
& +\sum_{n+2 \leq k+i \leq n+m+1} \sum_{i=1}^{n+j-1} Y_{n+j-i+1}^{(n+j)}\left(-U_{i}\right)_{k=2}^{-1} \sum_{k=1+n-1}^{m+i+1} X_{k}^{(i)}\left[B_{n, k+i-1-n}^{(\boldsymbol{d})}-F^{(\boldsymbol{d})}\right]
\end{aligned}
$$


then, we obtain that for $j=1,2, \ldots, m$,

$$
\begin{aligned}
G^{(\boldsymbol{d})}(n, j) & =g^{(\boldsymbol{d})}(n, j-1)-g^{(\boldsymbol{d})}(n, j) \\
& =R\left[W_{n, j-1}^{(\boldsymbol{d})}-W_{n, j}^{(\boldsymbol{d})}\right]-\left[V_{n, j-1}^{(\boldsymbol{d})}-V_{n, j}^{(\boldsymbol{d})}\right]+\left(1-G_{n+j}\right) \prod_{k=1}^{n+j-1} G_{k} .
\end{aligned}
$$

We can see that $G^{(\boldsymbol{d})}(n, j)$ quantifies the difference among two adjacent performance potentials $g^{(\boldsymbol{d})}(n, j)$ and $g^{(\boldsymbol{d})}(n, j-1)$. It measures the long-run effect on the average profit of the data center when the system state is changed from $(n, j-1)$ to $(n, j)$, which indicates the occurrence of a service completion event. From later discussion in Section 6 , we will see that $G^{(\boldsymbol{d})}(n, j)$ plays a fundamental role in the performance optimization of data centers and the sign of $G^{(\boldsymbol{d})}(n, j)+c$ directly determines the selection of decision actions, as shown in (49) later, where $c$ is defined as

$$
c=R-\frac{\left(P_{2, W}-P_{2, S}\right) C_{1}}{\mu_{2}} .
$$

To this end, we analyze how the service price impacts on $G^{(\boldsymbol{d})}(n, j)+c$ as follows.

Substituting (37) into the linear equation $G^{(\boldsymbol{d})}(n, j)+c=0$, we obtain

$$
R\left[W_{n, j-1}^{(\boldsymbol{d})}-W_{n, j}^{(\boldsymbol{d})}\right]-\left[V_{n, j-1}^{(\boldsymbol{d})}-V_{n, j}^{(\boldsymbol{d})}\right]+\left(1-G_{n+j}\right) \prod_{k=1}^{n+j-1} G_{k}+c=0 .
$$

Substituting (38) into the above equation, we obtain that the unique solution of the price $R$ in (39) is given by

$$
\Re_{n, j}^{(\boldsymbol{d})}=\frac{\left[V_{n, j-1}^{(\boldsymbol{d})}-V_{n, j}^{(\boldsymbol{d})}\right]-\left(1-G_{n+j}\right) \prod_{k=1}^{n+j-1} G_{k}+\frac{\left(P_{2, W}-P_{2, S}\right) C_{1}}{\mu_{2}}}{1+\left[W_{n, j-1}^{(\boldsymbol{d})}-W_{n, j}^{(\boldsymbol{d})}\right]} .
$$

It is easy to see that (a) if $R \geq \Re_{n, j}^{(\boldsymbol{d})}$, then $G^{(\boldsymbol{d})}(n, j)+c \geq 0$; and (b) if $R \leq \Re_{n, j}^{(\boldsymbol{d})}$, then $G^{(\boldsymbol{d})}(n, j)+c \leq 0$.

In the energy-efficient data center, we define two critical values, related to the service price, as

$$
R_{H}=\max _{\boldsymbol{d} \in \mathcal{D}}\left\{0, \Re_{n, 1}^{(\boldsymbol{d})}, \Re_{n, 2}^{(\boldsymbol{d})}, \ldots, \Re_{n, m}^{(\boldsymbol{d})}\right\}
$$

and

$$
R_{L}=\min _{\boldsymbol{d} \in \mathcal{D}}\left\{\Re_{n, 1}^{(\boldsymbol{d})}, \Re_{n, 2}^{(\boldsymbol{d})}, \ldots, \Re_{n, m}^{(\boldsymbol{d})}\right\} .
$$

The following proposition uses the two critical values related to the service price to provide a key condition whose purpose is to establish a sensitivity-based optimization 
framework of the energy-efficient data center in our later study. Also, this proposition will be useful in the next section for studying the monotonicity of the energy-efficient policies.

Proposition 2 (1) If $R \geq R_{H}$, then for any $\boldsymbol{d} \in \mathcal{D}$ and for each $j=1,2, \ldots, m$, we have

$$
G^{(\boldsymbol{d})}(n, j)+c \geq 0
$$

(2) If $0 \leq R \leq R_{L}$, then for any $\boldsymbol{d} \in \mathcal{D}$ and for each $j=1,2, \ldots, m$, we have

$$
G^{(\boldsymbol{d})}(n, j)+c \leq 0
$$

Proof: (1) For any $\boldsymbol{d} \in \mathcal{D}$ and for each $j=1,2, \ldots, m$, since $R \geq R_{H}$ and $R_{H}=$ $\max _{\boldsymbol{d} \in \mathcal{D}}\left\{0, \Re_{n, 1}^{(\boldsymbol{d})}, \Re_{n, 2}^{(\boldsymbol{d})}, \ldots, \Re_{n, m}^{(\boldsymbol{d})}\right\}$, we have

$$
R \geq \Re_{n, j}^{(\boldsymbol{d})},
$$

which clearly makes that $G^{(\boldsymbol{d})}(n, j)+c \geq 0$.

(2) For any $\boldsymbol{d} \in \mathcal{D}$ and for each $j=1,2, \ldots, m$, if $0 \leq R \leq R_{L}$, we have

$$
R \leq \Re_{n, j}^{(d)}
$$

this gives that $G^{(\boldsymbol{d})}(n, j)+c \leq 0$. This completes the proof.

\section{Monotonicity and Optimality}

In this section, we use the Poisson equation to derive a useful performance difference equation, and discuss the monotonicity and optimality of the long-run average profit of the energy-efficient data center with respect to the policies. Based on this, we give the optimal energy-efficient policy under some restrained service prices.

For any given policy $\boldsymbol{d} \in \mathcal{D}$, the policy-based continuous-time birth-death process $\left\{\mathbf{X}^{(\boldsymbol{d})}(t): t \geq 0\right\}$ with infinitesimal generator $\mathbf{B}^{(\boldsymbol{d})}$ given in (3) is irreducible, aperiodic and positive recurrent, hence the long-run average profit of the data center is given by

$$
\eta^{d}=\boldsymbol{\pi}^{(\boldsymbol{d})} \boldsymbol{f}^{(\boldsymbol{d})}
$$

and the Poisson equation is written as

$$
\mathbf{B}^{(\boldsymbol{d})} \boldsymbol{g}^{(\boldsymbol{d})}=\eta^{\boldsymbol{d}} \boldsymbol{e}-\boldsymbol{f}^{(\boldsymbol{d})}
$$


With a similar role played by state $(i, j)$, it is seen from (3) that the policy $\boldsymbol{d}$ directly affects not only the elements of the infinitesimal generator $\mathbf{B}^{(\boldsymbol{d})}$ but also the reward function $\boldsymbol{f}^{(\boldsymbol{d})}$. That is, if the policy $\boldsymbol{d}$ changes, then the infinitesimal generator $\mathbf{B}^{(\boldsymbol{d})}$ and the reward function $\boldsymbol{f}^{(\boldsymbol{d})}$ will have their corresponding changes. To express such a change mathematically, we take two different policies $\boldsymbol{d}$ and $\boldsymbol{d}^{\prime}$, both of which correspond to their infinitesimal generators $\mathbf{B}^{(\boldsymbol{d})}$ and $\mathbf{B}^{\left(\boldsymbol{d}^{\prime}\right)}$, and to their reward functions $\boldsymbol{f}^{(\boldsymbol{d})}$ and $\boldsymbol{f}^{\left(\boldsymbol{d}^{\prime}\right)}$.

The following lemma provides a useful equation for the difference $\eta^{\boldsymbol{d}^{\prime}}-\eta^{\boldsymbol{d}}$ of the longrun average performances $\eta^{\boldsymbol{d}}$ and $\eta^{\boldsymbol{d}^{\prime}}$ for any two policies $\boldsymbol{d}, \boldsymbol{d}^{\prime} \in \mathcal{D}$. The performance difference equation plays a key role in the sensitivity-based optimization theory. Note that the performance difference equation was given in Cao [5], while here we restate it with some simple discussion, for convenience of readers.

Lemma 1 For any two policies $\boldsymbol{d}, \boldsymbol{d}^{\prime} \in \mathcal{D}$, we have

$$
\eta^{\boldsymbol{d}^{\prime}}-\eta^{\boldsymbol{d}}=\boldsymbol{\pi}^{\left(\boldsymbol{d}^{\prime}\right)}\left[\left(\mathbf{B}^{\left(d^{\prime}\right)}-\mathbf{B}^{(\boldsymbol{d})}\right) \boldsymbol{g}^{(\boldsymbol{d})}+\left(\boldsymbol{f}^{\left(\boldsymbol{d}^{\prime}\right)}-\boldsymbol{f}^{(\boldsymbol{d})}\right)\right] .
$$

Proof: Note that $\boldsymbol{\pi}^{\left(d^{\prime}\right)} \mathbf{B}^{\left(d^{\prime}\right)}=\mathbf{0}, \mathbf{B}^{(d)} g^{(d)}=\eta^{d} \boldsymbol{e}-\boldsymbol{f}^{(d)}, \boldsymbol{\pi}^{\left(d^{\prime}\right)} \boldsymbol{e}=1$, we compute

$$
\begin{aligned}
& \boldsymbol{\pi}^{\left(d^{\prime}\right)}\left[\left(\mathbf{B}^{\left(d^{\prime}\right)}-\mathbf{B}^{(d)}\right) g^{(d)}+\left(\boldsymbol{f}^{\left(d^{\prime}\right)}-\boldsymbol{f}^{(d)}\right)\right] \\
& =-\boldsymbol{\pi}^{\left(d^{\prime}\right)} \cdot \mathbf{B}^{(d)} g^{(d)}+\boldsymbol{\pi}^{\left(d^{\prime}\right)} \boldsymbol{f}^{\left(d^{\prime}\right)}-\boldsymbol{\pi}^{\left(d^{\prime}\right)} \boldsymbol{f}^{(d)} \\
& =-\boldsymbol{\pi}^{\left(d^{\prime}\right)}\left[\eta^{d} e-\boldsymbol{f}^{(d)}\right]+\eta^{\boldsymbol{d}^{\prime}}-\boldsymbol{\pi}^{\left(\boldsymbol{d}^{\prime}\right)} \boldsymbol{f}^{(d)} \\
& =\eta^{\boldsymbol{d}^{\prime}}-\eta^{\boldsymbol{d}} .
\end{aligned}
$$

This completes the proof.

Now, we describe the first role played by the performance difference, in which we set up a partial order relation in the policy set $\mathcal{D}$ so that the optimal policy in $\mathcal{D}$ can be found numerically. Based on the performance difference $\eta^{\boldsymbol{d}^{\prime}}-\eta^{\boldsymbol{d}}$ for any two policies $\boldsymbol{d}, \boldsymbol{d}^{\prime} \in \mathcal{D}$, we can set up a partial order in the policy set $\mathcal{D}$ as follows. We write $\boldsymbol{d}^{\prime} \succ \boldsymbol{d}$ if $\eta^{\boldsymbol{d}^{\prime}}>\eta^{\boldsymbol{d}}$; $\boldsymbol{d}^{\prime} \approx \boldsymbol{d}$ if $\eta^{\boldsymbol{d}^{\prime}}=\eta^{\boldsymbol{d}} ; \boldsymbol{d}^{\prime} \prec \boldsymbol{d}$ if $\eta^{\boldsymbol{d}^{\prime}}<\eta^{\boldsymbol{d}}$. Also, we write $\boldsymbol{d}^{\prime} \succeq \boldsymbol{d}$ if $\eta^{\boldsymbol{d}^{\prime}} \geq \eta^{\boldsymbol{d}} ; \boldsymbol{d}^{\prime} \preceq \boldsymbol{d}$ if $\eta^{\boldsymbol{d}^{\prime}} \leq \eta^{\boldsymbol{d}}$. By using this partial order, our research target is to find an optimal policy $\boldsymbol{d}^{*} \in \mathcal{D}$ such that $\boldsymbol{d}^{*} \succeq \boldsymbol{d}$ for any policy $\boldsymbol{d} \in \mathcal{D}$, or

$$
\boldsymbol{d}^{*}=\underset{\boldsymbol{d} \in \mathcal{D}}{\arg \max }\left\{\eta^{\boldsymbol{d}}\right\}
$$

Note that the policy set $\mathcal{D}$ and the state space $\boldsymbol{\Omega}$ are both finite, thus an enumeration method is feasible for finding the optimal energy-efficient policy $\boldsymbol{d}^{*}$ in the policy set $\mathcal{D}$. 
Since $\boldsymbol{d}=\left(0,0, \ldots, 0 ; d_{n, 1}, d_{n, 2}, \ldots, d_{n, m}\right)$ and $d_{n, j} \in\{0,1, \ldots, m\}$, it is seen that the policy set $\mathcal{D}$ contains $(m+1)^{m}$ elements so that the enumeration method will require a huge computation workload. However, our following work can greatly reduce the optimization complexity by means of the sensitivity-based optimization theory.

Now, we discuss the monotonicity of the long-run average profit $\eta^{d}$ with respect to a decision element $d_{n, j}$ of any policy $\boldsymbol{d} \in \mathcal{D}$, for $d_{n, j}=0,1, \ldots, m$. This result is derived by the following two theorems, in which we show that for any policy $\boldsymbol{d} \in \mathcal{D}$ and for each $j=1,2, \ldots, m$, the long-run average profit $\eta^{\boldsymbol{d}}$ is unimodal with respect to each decision element $d_{n, j} \in\{0,1, \ldots, m\}$.

Theorem 3 For any policy $\boldsymbol{d} \in \mathcal{D}$ and for each $j=1,2, \ldots, m$, the long-run average profit $\eta^{\boldsymbol{d}}$ is linearly decreasing with respect to each decision element $d_{n, j}$, where $d_{n, j} \in$ $\{j, j+1, \ldots, m\}$.

Proof: For each $j=1,2, \ldots, m$, we consider two interrelated policies $\boldsymbol{d}, \boldsymbol{d}^{\prime} \in \mathcal{D}$ as follows.

$$
\begin{aligned}
\boldsymbol{d} & =\left(0,0, \ldots, 0 ; d_{n, 1}, d_{n, 2}, \ldots, d_{n, j-1}, \underline{d_{n, j}}, d_{n, j+1}, \ldots, d_{n, m}\right) \\
\boldsymbol{d}^{\prime} & =\left(0,0, \ldots, 0 ; d_{n, 1}, d_{n, 2}, \ldots, d_{n, j-1} \underline{j,} d_{n, j+1}, \ldots, d_{n, m}\right)
\end{aligned}
$$

where $d_{n, j}>j$. It is seen that the two policies $\boldsymbol{d}, \boldsymbol{d}^{\prime}$ have one difference only between their corresponding decision elements $d_{n, j}$ and $j$. In this case, it is seen from Theorem 1 that $\mathbf{B}^{(\boldsymbol{d})}=\mathbf{B}^{\left(\boldsymbol{d}^{\prime}\right)}$ and $\boldsymbol{\pi}^{(\boldsymbol{d})}=\boldsymbol{\pi}^{\left(\boldsymbol{d}^{\prime}\right)}$. Also, it is easy to check from (13) to (17) that

$$
\boldsymbol{f}^{(\boldsymbol{d})}-\boldsymbol{f}^{\left(\boldsymbol{d}^{\prime}\right)}=\left(0,0, \ldots, 0 ; 0,0, \ldots, 0,-\left(d_{n, j}-j\right)\left(P_{2, W}-P_{2, S}\right) C_{1}, 0, \ldots, 0\right)^{T} .
$$

Thus, it follows from Lemma 1 that

$$
\begin{aligned}
\eta^{\boldsymbol{d}}-\eta^{\boldsymbol{d}^{\prime}} & =\boldsymbol{\pi}^{(\boldsymbol{d})}\left[\left(\mathbf{B}^{(\boldsymbol{d})}-\mathbf{B}^{\left(\boldsymbol{d}^{\prime}\right)}\right) \boldsymbol{g}^{\left(\boldsymbol{d}^{\prime}\right)}+\left(\boldsymbol{f}^{(\boldsymbol{d})}-\boldsymbol{f}^{\left(\boldsymbol{d}^{\prime}\right)}\right)\right] \\
& =-\pi^{(\boldsymbol{d})}(n, j)\left(d_{n, j}-j\right)\left(P_{2, W}-P_{2, S}\right) C_{1}
\end{aligned}
$$

or

$$
\eta^{\boldsymbol{d}}=\eta^{\boldsymbol{d}^{\prime}}-\pi^{(\boldsymbol{d})}(n, j)\left(d_{n, j}-j\right)\left(P_{2, W}-P_{2, S}\right) C_{1} .
$$

Since $\boldsymbol{\pi}^{(\boldsymbol{d})}=\boldsymbol{\pi}^{\left(\boldsymbol{d}^{\prime}\right)}$ by Theorem 1, it is easy to see that $\pi^{(\boldsymbol{d})}(n, j)=\pi^{\left(\boldsymbol{d}^{\prime}\right)}(n, j)$ can be determined by $d_{n, j}^{\prime}=j$. This indicates that $\pi^{(\boldsymbol{d})}(n, j)$ is irrelevant to the decision element $d_{n, j}$. Again, note that $\eta^{\boldsymbol{d}^{\prime}}$ is irrelevant to the decision element $d_{n, j}$, and $P_{2, W}-P_{2, S}$ and $C_{1}$ 
are two positive constants. Thus, it is easy to see from (46) that the long-run average profit $\eta^{\boldsymbol{d}}$ is linearly decreasing with respect to each decision element $d_{n, j} \in\{j, j+1, \ldots, m\}$. This completes the proof.

In what follows we discuss the left half part of the unimodal structure of the long-run average profit $\eta^{\boldsymbol{d}}$ with respect to each decision element $d_{n, j} \in\{0,1, \ldots, j\}$. Compared to the analysis of its right half part in Theorem 3, our discussion for the left half part is a little bit complicated.

Let the optimal energy-efficient policy $\boldsymbol{d}^{*}=\underset{\boldsymbol{d} \in \mathcal{D}}{\arg \max }\left\{\eta^{\boldsymbol{d}}\right\}$ be

$$
\boldsymbol{d}^{*}=\left(0,0, \ldots, 0 ; d_{n, 1}^{*}, d_{n, 2}^{*}, \ldots, d_{n, m}^{*}\right)
$$

Then, it is seen from Theorem 3 that

$$
\begin{gathered}
d_{n, 1}^{*} \in\{0,1\} ; \\
\vdots \\
d_{n, j}^{*} \in\{0,1, \ldots, j\} ; \\
\vdots \\
d_{n, m}^{*} \in\{0,1, \ldots, m\} .
\end{gathered}
$$

Thus, Theorem 3 makes the area of finding the optimal energy-efficient policy $\boldsymbol{d}^{*}$ from a large set $\{0,1, \ldots, m\}^{m}$ to a shrunken area $\{0,1\} \times\{0,1,2\} \times \cdots \times\{0,1, \ldots, m\}$.

To find the optimal energy-efficient policy $\boldsymbol{d}^{*}$, we consider two energy-efficient policies with an interrelated structure as follows.

$$
\begin{aligned}
\boldsymbol{d} & =\left(0,0, \ldots, 0 ; d_{n, 1}, \ldots, d_{n, j-1}, \underline{d_{n, j}}, d_{n, j+1}, \ldots, d_{n, m}\right) \\
\boldsymbol{d}^{\prime} & =\left(0,0, \ldots, 0 ; d_{n, 1}, \ldots, d_{n, j-1}, \underline{d_{n, j}^{\prime}}, d_{n, j+1}, \ldots, d_{n, m}\right)
\end{aligned}
$$

where $d_{n, j}^{\prime}>d_{n, j}$, and $d_{n, j}, d_{n, j}^{\prime} \in\{1,2, \ldots, j\}$. It is easy to check from (3) that

$$
\mathbf{B}^{\left(\boldsymbol{d}^{\prime}\right)}-\mathbf{B}^{(\boldsymbol{d})}=\left(\begin{array}{ccccccc}
0 & & & & & & \\
0 & \ddots & & & & & \\
& \ddots & 0 & & & & \\
& & \left(d_{n, j}^{\prime}-d_{n, j}\right) \mu_{2} & -\left(d_{n, j}^{\prime}-d_{n, j}\right) \mu_{2} & & & \\
& & 0 & 0 & & \\
& & & & \ddots & \ddots & \\
& & & & 0 & 0
\end{array}\right) .
$$


On the other hand, from the reward functions given in (13) to (15), it is seen that for $j=1,2, \ldots, m$, and $d_{n, j}=0,1, \ldots, m$,

$$
\begin{aligned}
f^{(\boldsymbol{d})}(n, j)= & {\left[R \mu_{2}-\left(P_{2, W}-P_{2, S}\right) C_{1}\right] d_{n, j} } \\
& +R n \mu_{1}-\left(n P_{1, W}-m P_{2, S}\right) C_{1}-\left[n C_{2}^{(1)}+j C_{2}^{(2)}\right]-n \mu_{1} 1_{\{j>0\}} C_{3}-\lambda 1_{\{i=n, j=m\}} C_{4}
\end{aligned}
$$

and

$$
\begin{aligned}
f^{\left(\boldsymbol{d}^{\prime}\right)}(n, j)= & {\left[R \mu_{2}-\left(P_{2, W}-P_{2, S}\right) C_{1}\right] d_{n, j}^{\prime} } \\
& +R n \mu_{1}-\left(n P_{1, W}-m P_{2, S}\right) C_{1}-\left[n C_{2}^{(1)}+j C_{2}^{(2)}\right]-n \mu_{1} 1_{\{j>0\}} C_{3}-\lambda 1_{\{i=n, j=m\}} C_{4} .
\end{aligned}
$$

Hence, we have

$$
\boldsymbol{f}^{\left(\boldsymbol{d}^{\prime}\right)}-\boldsymbol{f}^{(\boldsymbol{d})}=\left(0,0, \ldots, 0, \mu_{2} c\left(d_{n, j}^{\prime}-d_{n, j}\right), 0, \ldots, 0\right)^{T}
$$

We write

$$
\left.\eta^{\boldsymbol{d}}\right|_{d_{n, j}=k}=\left.\left.\boldsymbol{\pi}^{(\boldsymbol{d})}\right|_{d_{n, j}=k} \cdot \boldsymbol{f}^{(\boldsymbol{d})}\right|_{d_{n, j}=k}
$$

The following theorem discusses the left half part of the unimodal structure of the long-run average profit $\eta^{\boldsymbol{d}}$ with respect to each decision element $d_{n, j} \in\{0,1, \ldots, m\}$.

Theorem 4 If $R \geq R_{H}$, then for any policy $\boldsymbol{d} \in \mathcal{D}$ and for each $j=1,2, \ldots, m$, the long-run average profit $\eta^{\boldsymbol{d}}$ is strictly monotone increasing with respect to each decision element $d_{n, j}$, where $d_{n, j} \in\{0,1, \ldots, j\}$.

Proof: For each $j=1,2, \ldots, m$, we consider two energy-efficient policies with an interrelated structure as follows.

$$
\begin{aligned}
\boldsymbol{d} & =\left(0,0, \ldots, 0 ; d_{n, 1}, \ldots, d_{n, j-1}, \underline{d_{n, j}}, d_{n, j+1}, \ldots, d_{n, m}\right) \\
\boldsymbol{d}^{\prime} & =\left(0,0, \ldots, 0 ; d_{n, 1}, \ldots, d_{n, j-1}, \underline{d_{n, j}^{\prime}}, d_{n, j+1}, \ldots, d_{n, m}\right)
\end{aligned}
$$

where $d_{n, j}^{\prime}>d_{n, j}$, and $d_{n, j}, d_{n, j}^{\prime} \in\{0,1, \ldots, j\}$. Applying Lemma 1, it follows from (47) and (48) that

$$
\begin{aligned}
\eta^{\boldsymbol{d}^{\prime}}-\eta^{\boldsymbol{d}} & =\boldsymbol{\pi}^{\left(\boldsymbol{d}^{\prime}\right)}\left[\left(\mathbf{B}^{\left(\boldsymbol{d}^{\prime}\right)}-\mathbf{B}^{(\boldsymbol{d})}\right) \boldsymbol{g}^{(\boldsymbol{d})}+\left(\boldsymbol{f}^{\left(\boldsymbol{d}^{\prime}\right)}-\boldsymbol{f}^{(\boldsymbol{d})}\right)\right] \\
& =\mu_{2} \pi^{\left(\boldsymbol{d}^{\prime}\right)}(n, j)\left(d_{n, j}^{\prime}-d_{n, j}\right)\left[g^{(\boldsymbol{d})}(n, j-1)-g^{(\boldsymbol{d})}(n, j)+c\right] \\
& =\mu_{2} \pi^{\left(\boldsymbol{d}^{\prime}\right)}(n, j)\left(d_{n, j}^{\prime}-d_{n, j}\right)\left[G^{(\boldsymbol{d})}(n, j)+c\right]
\end{aligned}
$$


where $G^{(\boldsymbol{d})}(n, j)=g^{(\boldsymbol{d})}(n, j-1)-g^{(\boldsymbol{d})}(n, j)$. If $R \geq R_{H}$, then it is seen from Proposition 2 that $G^{(\boldsymbol{d})}(n, j)+c \geq 0$. Thus, we obtain that for the two policies $\boldsymbol{d}, \boldsymbol{d}^{\prime} \in \mathcal{D}$ with $d_{n, j}^{\prime}>d_{n, j}$ and $d_{n, j}, d_{n, j}^{\prime} \in\{0,1, \ldots, j\}$,

$$
\eta^{\boldsymbol{d}^{\prime}}>\eta^{\boldsymbol{d}}
$$

This shows that

$$
\left.\eta^{\boldsymbol{d}}\right|_{d_{n, j}=1}<\left.\eta^{\boldsymbol{d}}\right|_{d_{n, j}=2}<\cdots<\left.\eta^{\boldsymbol{d}}\right|_{d_{n, j}=m-1}<\left.\eta^{\boldsymbol{d}}\right|_{d_{n, j}=m}
$$

This completes the proof.

When $R \geq R_{H}$, we use Figure 2 to provide an intuitive summary for the main results given in Theorems 3 and 4 . In the right half part of Figure 2,

$$
\eta^{\boldsymbol{d}}=\eta^{\boldsymbol{d}^{\prime}}-\pi^{(\boldsymbol{d})}(n, j)\left(d_{n, j}-j\right)\left(P_{2, W}-P_{2, S}\right) C_{1}
$$

shows that $\eta^{d}$ is a linear function of the decision element $d_{n, j}$. By contrast, in the right half part of Figure 2, we need to first introduce a restrictive condition: $R \geq R_{H}$, under which

$$
\eta^{\boldsymbol{d}^{\prime}}-\eta^{\boldsymbol{d}}=\mu_{2} \pi^{\left(\boldsymbol{d}^{\prime}\right)}(n, j)\left(d_{n, j}^{\prime}-d_{n, j}\right)\left[G^{(\boldsymbol{d})}(n, j)+c\right] .
$$

Let $d_{n, j}^{\prime}=j$. Then,

$$
\eta^{\boldsymbol{d}}=\eta^{\boldsymbol{d}^{\prime}}-\mu_{2} \pi^{\left(\boldsymbol{d}^{\prime}\right)}(n, j)\left(j-d_{n, j}\right)\left[G^{(\boldsymbol{d})}(n, j)+c\right] .
$$

Since $G^{(\boldsymbol{d})}(n, j)$ depends on the decision element $d_{n, j}$, it is clear that $\eta^{\boldsymbol{d}}$ is a nonlinear function of the decision element $d_{n, j}$.

Theorem 5 If $0 \leq R \leq R_{L}$, then for any $\boldsymbol{d} \in \mathcal{D}$ and for each $j=1,2, \ldots, m$, the longrun average profit $\eta^{\boldsymbol{d}}$ is strictly monotone decreasing with respect to each decision element $d_{n, j}$, where $d_{n, j} \in\{0,1, \ldots, j\}$.

Proof: This proof is similar to the proof of Theorem 4. For each $j=1,2, \ldots, m$, we consider two energy-efficient policies with an interrelated structure as follows.

$$
\begin{aligned}
\boldsymbol{d} & =\left(0,0, \ldots, 0 ; d_{n, 1}, \ldots, d_{n, j-1}, \underline{d_{n, j}}, d_{n, j+1}, \ldots, d_{n, m}\right) \\
\boldsymbol{d}^{\prime} & =\left(0,0, \ldots, 0 ; d_{n, 1}, \ldots, d_{n, j-1}, \underline{d_{n, j}^{\prime}}, d_{n, j+1}, \ldots, d_{n, m}\right)
\end{aligned}
$$




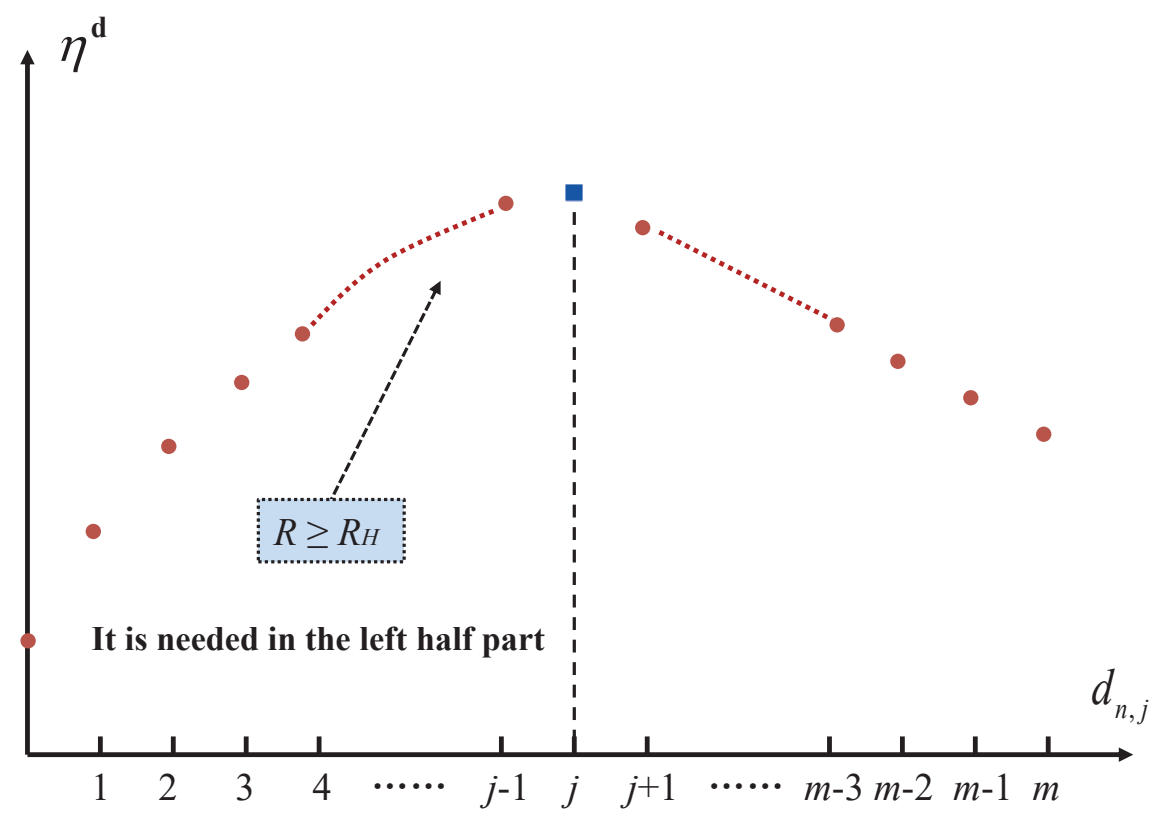

Figure 2: The unimodal structure of the long-run average profit.

where $d_{n, j}^{\prime}>d_{n, j}$, and $d_{n, j}, d_{n, j}^{\prime} \in\{0,1, \ldots, j\}$. It is clear that

$$
\eta^{\boldsymbol{d}^{\prime}}-\eta^{\boldsymbol{d}}=\mu_{2} \pi^{\left(\boldsymbol{d}^{\prime}\right)}(n, j)\left(d_{n, j}^{\prime}-d_{n, j}\right)\left[G^{(\boldsymbol{d})}(n, j)+c\right] .
$$

If $0 \leq R \leq R_{L}$, then it is seen from Proposition 2 that for any $\boldsymbol{d} \in \mathcal{D}$ and for each $j=1,2, \ldots, m, G^{(\boldsymbol{d})}(n, j)+c \leq 0$. Thus, we obtain that for the two policies $\boldsymbol{d}, \boldsymbol{d}^{\prime} \in \mathcal{D}$ with $d_{n, j}^{\prime}>d_{n, j}$ and $d_{n, j}, d_{n, j}^{\prime} \in\{0,1, \ldots, j\}$,

$$
\eta^{\boldsymbol{d}^{\prime}}<\eta^{\boldsymbol{d}}
$$

This shows that

$$
\left.\eta^{\boldsymbol{d}}\right|_{d_{n, j}=1}>\left.\eta^{\boldsymbol{d}}\right|_{d_{n, j}=2}>\cdots>\left.\eta^{\boldsymbol{d}}\right|_{d_{n, j}=m-1}>\left.\eta^{\boldsymbol{d}}\right|_{d_{n, j}=m}
$$

This completes the proof.

When $0 \leq R \leq R_{L}$, we use Figure 3 to provide an intuitive summary for the main results given in Theorems 3 and 5 ,

The following theorem establishes the optimal energy-efficient policy $\boldsymbol{d}^{*}$ in the data center, and also computes the maximal long-run average profit.

Theorem 6 The optimal energy-efficient policy $\boldsymbol{d}^{*}$ and the maximal long-run average profit $\eta^{\boldsymbol{d}^{*}}$ can be determined in the following two different cases: 


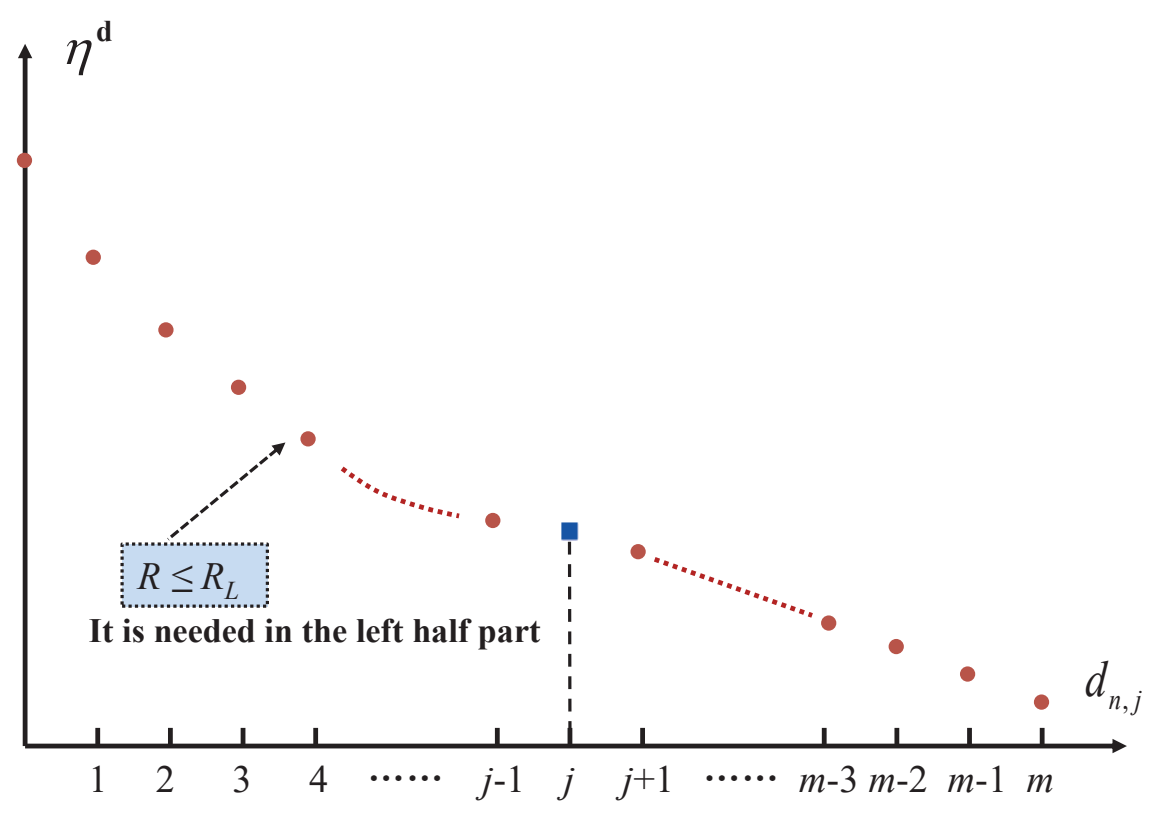

Figure 3: The decreasing structure of the long-run average profit.

(a) If $R \geq R_{H}$, then

$$
\boldsymbol{d}^{*}=(0,0, \ldots, 0 ; 1,2, \ldots, m)
$$

and

$$
\begin{aligned}
& \eta^{\boldsymbol{d}^{*}}=\sum_{i=0}^{n} \frac{\frac{\lambda^{i}}{i ! \mu_{1}^{i}}}{\sum_{i=0}^{n} \frac{\lambda^{i}}{i ! \mu_{1}^{i}}+\frac{\lambda^{n}}{n ! \mu_{1}^{n}} \sum_{j=1}^{m} \frac{\lambda^{j}}{\prod_{i=1}^{j}\left(n \mu_{1}+i \mu_{2}\right)}}\left[R i \mu_{1}-\left(n P_{1, W}+m P_{2, S}\right) C_{1}-i C_{2}^{(1)}\right] \\
& \frac{\lambda^{n}}{n ! \mu_{1}^{n}} \frac{\lambda^{j}}{\prod_{i=1}^{j}\left(n \mu_{1}+i \mu_{2}\right)} \\
& +\sum_{j=1}^{m} \frac{n ! \mu_{1}^{n} \prod_{i=1}^{j}\left(n \mu_{1}+i \mu_{2}\right)}{\sum_{i=0}^{n} \frac{\lambda^{i}}{i ! \mu_{1}^{i}}+\frac{\lambda^{n}}{n ! \mu_{1}^{n}} \sum_{j=1}^{m} \frac{\lambda^{j}}{\prod_{i=1}^{j}\left(n \mu_{1}+i \mu_{2}\right)}}\left\{R\left(n \mu_{1}+j \mu_{2}\right)-\left[n C_{2}^{(1)}+j C_{2}^{(2)}\right]\right. \\
& \left.-\left[n P_{1, W}+j P_{2, W}+(m-j) P_{2, S}\right] C_{1}-n \mu_{1} 1_{\{j>0\}} C_{3}-\lambda 1_{\{i=n, j=m\}} C_{4}\right\} .
\end{aligned}
$$

(b) If $0 \leq R \leq R_{L}$, then

$$
\boldsymbol{d}^{*}=(0,0, \ldots, 0 ; 0,0, \ldots, 0)
$$


and

$$
\begin{aligned}
\eta^{\boldsymbol{d}^{*}}= & \sum_{i=0}^{n} \frac{\frac{\lambda^{i}}{i ! \mu_{1}^{i}}}{\sum_{i=0}^{n} \frac{\lambda^{i}}{i ! \mu_{1}^{i}}+\frac{\lambda^{n}}{n ! \mu_{1}^{n}} \sum_{j=1}^{m} \frac{\lambda^{j}}{\left(n \mu_{1}\right)^{j}}}\left[R i \mu_{1}-\left(n P_{1, W}+m P_{2, S}\right) C_{1}-i C_{2}^{(1)}\right] \\
& +\sum_{j=1}^{m} \frac{\frac{\lambda^{n}}{n ! \mu_{1}^{n}} \frac{\lambda^{j}}{\left(n \mu_{1}\right)^{j}}}{\sum_{i=0}^{n} \frac{\lambda^{i}}{i ! \mu_{1}^{i}}+\frac{\lambda^{n}}{n ! \mu_{1}^{n}} \sum_{j=1}^{m} \frac{\lambda^{j}}{\left(n \mu_{1}\right)^{j}}}\left\{R n \mu_{1}-\left[n P_{1, W}+m P_{2, S}\right] C_{1}\right. \\
& \left.-\left[n C_{2}^{(1)}+j C_{2}^{(2)}\right]-n \mu_{1} 1_{\{j>0\}} C_{3}-\lambda 1_{\{i=n, j=m\}} C_{4}\right\} .
\end{aligned}
$$

Proof: (a) For the optimal energy-efficient policy $\boldsymbol{d}^{*}=(0,0, \ldots, 0 ; 1,2, \ldots, m)$, it is clear that $d_{n, j}^{*}=j$ and $d_{n, j}^{*} \wedge j=j$. Thus, it follows from (5), (6) and (17) that

$$
\xi_{i, 0}=\frac{\lambda^{i}}{i ! \mu_{1}^{i}}, \quad i=0,1, \ldots, n,
$$

and

$$
\begin{gathered}
\xi_{n, j}^{\left(\boldsymbol{d}^{*}\right)}=\frac{\lambda^{n}}{n ! \mu_{1}^{n}} \frac{\lambda^{j}}{\prod_{i=1}^{j}\left(n \mu_{1}+i \mu_{2}\right)}, \quad j=1,2, \ldots, m, \\
b^{\left(\boldsymbol{d}^{*}\right)}=\sum_{i=0}^{n} \frac{\lambda^{i}}{i ! \mu_{1}^{i}}+\frac{\lambda^{n}}{n ! \mu_{1}^{n}} \sum_{j=1}^{m} \frac{\lambda^{j}}{\prod_{i=1}^{j}\left(n \mu_{1}+i \mu_{2}\right)} .
\end{gathered}
$$

It follows from (8) and (9) that for $i=0,1, \ldots, n$,

$$
\pi^{\left(\boldsymbol{d}^{*}\right)}(i, 0)=\frac{\frac{\lambda^{i}}{i ! \mu_{1}^{i}}}{\sum_{i=0}^{n} \frac{\lambda^{i}}{i ! \mu_{1}^{i}}+\frac{\lambda^{n}}{n ! \mu_{1}^{n}} \sum_{j=1}^{m} \frac{\lambda^{j}}{\prod_{i=1}^{j}\left(n \mu_{1}+i \mu_{2}\right)}},
$$

and for $j=1,2, \ldots, m$,

$$
\pi^{\left(\boldsymbol{d}^{*}\right)}(n, j)=\frac{\frac{\lambda^{n}}{n ! \mu_{1}^{n}} \frac{\lambda^{j}}{\prod_{i=1}^{j}\left(n \mu_{1}+i \mu_{2}\right)}}{\sum_{i=0}^{n} \frac{\lambda^{i}}{i ! \mu_{1}^{i}}+\frac{\lambda^{n}}{n ! \mu_{1}^{n}} \sum_{j=1}^{m} \frac{\lambda^{j}}{\prod_{i=1}^{j}\left(n \mu_{1}+i \mu_{2}\right)}} .
$$

At the same time, from (11) to (16) we obtain that for $i=0,1, \ldots, n$,

$$
f(i, 0)=R i \mu_{1}-\left(n P_{1, W}+m P_{2, S}\right) C_{1}-i C_{2}^{(1)},
$$


and $j=1,2, \ldots, m$,

$$
\begin{aligned}
f^{\left(\boldsymbol{d}^{*}\right)}(n, j)= & R\left(n \mu_{1}+j \mu_{2}\right)-\left[n P_{1, W}+j P_{2, W}+(m-j) P_{2, S}\right] C_{1} \\
& -\left[n C_{2}^{(1)}+j C_{2}^{(2)}\right]-n \mu_{1} 1_{\{j>0\}} C_{3}-\lambda 1_{\{i=n, j=m\}} C_{4} .
\end{aligned}
$$

Thus, we obtain

$$
\eta^{\boldsymbol{d}^{*}}=\sum_{i=0}^{n} \pi^{\left(\boldsymbol{d}^{*}\right)}(i, 0) f(i, 0)+\sum_{j=1}^{m} \pi^{\left(\boldsymbol{d}^{*}\right)}(n, j) f^{\left(\boldsymbol{d}^{*}\right)}(n, j) .
$$

A simple computation directly derives our desired result.

(b) For the optimal policy $\boldsymbol{d}^{*}=(0,0, \ldots, 0 ; 0,0, \ldots, 0)$, it is clear that $d_{n, j}^{*}=0$ so that $d_{n, j}^{*} \wedge j=0$. A similar analysis to that in (a) can lead to our desired result. This completes the proof.

Remark 5 The results of Theorem 6 are intuitive due to the fact that when the service price is suitably high, the number of working servers is equal to the number of waiting jobs in Group 2; while when the service price is lower, each server opened at the work state will pay a high energy consumption cost but receive a low revenue, thus the profit cannot increase and all the servers in Group 2 would like to be at the sleep state.

When the price $R_{L}<R<R_{H}$, we can further derive the following theorem about the monotonicity of $\eta^{\boldsymbol{d}}$ with respect to the decision element $d_{n, j}$.

Theorem 7 If $R_{L}<R<R_{H}$, then the long-run average profit $\eta^{\boldsymbol{d}}$ is monotone (either increasing or decreasing) with respect to the decision element $d_{n, j}$, where $j=1,2, \ldots, m$ and $d_{n, j} \in\{0,1, \ldots, j\}$.

Proof: Similar to the first part of the proof for Theorem 4, we consider any two energy-efficient policies with an interrelated structure as follows.

$$
\begin{aligned}
\boldsymbol{d} & =\left(0,0, \ldots, 0 ; d_{n, 1}, \ldots, d_{n, j-1}, \underline{d_{n, j}}, d_{n, j+1}, \ldots, d_{n, m}\right) \\
\boldsymbol{d}^{\prime} & =\left(0,0, \ldots, 0 ; d_{n, 1}, \ldots, d_{n, j-1}, \underline{d_{n, j}^{\prime}}, d_{n, j+1}, \ldots, d_{n, m}\right)
\end{aligned}
$$

where $d_{n, j}, d_{n, j}^{\prime} \in\{0,1, \ldots, j\}$. Applying Lemma 1, we obtain

$$
\eta^{\boldsymbol{d}^{\prime}}-\eta^{\boldsymbol{d}}=\mu_{2} \pi^{\left(\boldsymbol{d}^{\prime}\right)}(n, j)\left(d_{n, j}^{\prime}-d_{n, j}\right)\left[G^{(\boldsymbol{d})}(n, j)+c\right] .
$$


On the other hand, we can similarly obtain the following difference equation

$$
\eta^{\boldsymbol{d}}-\eta^{\boldsymbol{d}^{\prime}}=\mu_{2} \pi^{(\boldsymbol{d})}(n, j)\left(d_{n, j}-d_{n, j}^{\prime}\right)\left[G^{\left(\boldsymbol{d}^{\prime}\right)}(n, j)+c\right] .
$$

By summing (50) and (51), we have

$\mu_{2} \pi^{\left(\boldsymbol{d}^{\prime}\right)}(n, j)\left(d_{n, j}^{\prime}-d_{n, j}\right)\left[G^{(\boldsymbol{d})}(n, j)+c\right]+\mu_{2} \pi^{(\boldsymbol{d})}(n, j)\left(d_{n, j}-d_{n, j}^{\prime}\right)\left[G^{\left(\boldsymbol{d}^{\prime}\right)}(n, j)+c\right]=0$.

We can directly derive

$$
\pi^{\left(\boldsymbol{d}^{\prime}\right)}(n, j)\left[G^{(\boldsymbol{d})}(n, j)+c\right]=\pi^{(\boldsymbol{d})}(n, j)\left[G^{\left(\boldsymbol{d}^{\prime}\right)}(n, j)+c\right] .
$$

Therefore, we have the sign conservation equation

$$
\frac{G^{(\boldsymbol{d})}(n, j)+c}{G^{\left(\boldsymbol{d}^{\prime}\right)}(n, j)+c}=\frac{\pi^{(\boldsymbol{d})}(n, j)}{\pi^{\left(\boldsymbol{d}^{\prime}\right)}(n, j)}>0
$$

The above equation means that the sign of $G^{(\boldsymbol{d})}(n, j)+c$ and $G^{\left(\boldsymbol{d}^{\prime}\right)}(n, j)+c$ are always identical when a particular decision element $d_{n, j}$ is changed to any $d_{n, j}^{\prime}$. With the sign conservation equation (52) and the performance difference equation (51), we can directly derive that the long-run average profit $\eta^{\boldsymbol{d}}$ is monotone with respect to $d_{n, j}$. This completes the proof.

Based on Theorems 4, 5, and7, we can directly derive that the optimal decision element $d_{n, j}^{*}$ has the bang-bang control form, no matter what the value of $R$ will be.

Corollary 1 The optimal decision element $d_{n, j}^{*}$ is either 0 or $j$, i.e., the bang-bang control is optimal.

With Corollary 1, we should either keep all servers sleep or turn on the servers such that the number of working servers equals the number of waiting jobs in Group 2. We can see that the search space of $d_{n, j}$ can be reduced from $\{0,1, \ldots, j\}$ to a 2-element set $\{0, j\}$, which is a significant reduction of optimization complexity. The form of the bang-bang control is also very simple and it is easy to adopt in practice, while the optimality of the bang-bang control guarantees the performance confidence of such simple forms of control.

\section{Threshold Energy-Efficient Policy}

We have proved the optimality of the bang-bang control, no matter what the value of $R$ will be. In practice, threshold-type policy is another category of policies which 
also have a very simple form and are widely adopted in many practical systems. In this section, we focus our study on the threshold-type policy, although its optimality is not yet proved rigorously in our problem. We use the Poisson equation to study a class of threshold energy-efficient policies, and obtain the necessary condition of the optimal threshold energy-efficient policy.

Now, we introduce an interesting subset of the policy set $\mathcal{D}$ as follows. To this end, for $\theta=1,2, \ldots, m+1$, we write $\boldsymbol{d}_{\theta}$ as an energy-efficient policy $\boldsymbol{d}$ with $d_{n, j}=0$ if $1 \leq j \leq \theta-1$ and $d_{n, j}=j$ if $\theta \leq j \leq m$, i.e.,

$$
\boldsymbol{d}_{\theta} \stackrel{\text { def }}{=}(0,0, \ldots, 0 ; \underbrace{0,0, \ldots, 0}_{\theta-1 \text { zeros }}, \theta, \theta+1, \ldots, m) \text {. }
$$

Let

$$
\mathcal{D}^{\Delta} \stackrel{\text { def }}{=}\left\{\boldsymbol{d}_{\theta}: \theta=1,2, \ldots, m+1\right\} .
$$

Then,

$$
\mathcal{D}^{\Delta}=\{(0,0, \ldots, 0 ; \underbrace{0,0, \ldots, 0}_{\theta-1 \text { zeros }}, \theta, \theta+1, \ldots, m): \theta=1,2, \ldots, m+1\} .
$$

It is easy to see that $\mathcal{D}^{\Delta} \subset \mathcal{D}$.

For a policy $\boldsymbol{d}_{\theta}$, it is clear that if $1 \leq j \leq \theta-1$, then $d_{n, j}=0$ and $d_{n, j} \wedge j=0$; and if $\theta \leq j \leq m$, then $d_{n, j}=j$ and $d_{n, j} \wedge j=j$. Thus, it follows from (15), (66) and (7) that

$$
\xi_{i, 0}=\frac{\lambda^{i}}{i ! \mu_{1}^{i}}, \quad i=0,1, \ldots, n,
$$

and

$$
\begin{gathered}
\xi_{n, j}^{\left(\boldsymbol{d}_{\theta}\right)}=\frac{\lambda^{n}}{n ! \mu_{1}^{n}} \frac{\lambda^{j}}{\left(n \mu_{1}\right)^{j}}, \quad j=1,2, \ldots, \theta-1, \\
\xi_{n, j}^{\left(\boldsymbol{d}_{\theta}\right)}=\frac{\lambda^{n}}{n ! \mu_{1}^{n}} \frac{\lambda^{\theta-1}}{\left(n \mu_{1}\right)^{\theta-1}} \frac{\lambda^{j-\theta+1}}{\prod_{i=\theta}^{j}\left(n \mu_{1}+i \mu_{2}\right)}, \quad j=\theta, \theta+1, \ldots, m, \\
b^{\left(\boldsymbol{d}_{\theta}\right)}=\sum_{i=0}^{n} \frac{\lambda^{i}}{i ! \mu_{1}^{i}}+\frac{\lambda^{n}}{n ! \mu_{1}^{n}} \sum_{j=1}^{\theta-1} \frac{\lambda^{j}}{\left(n \mu_{1}\right)^{j}}+\frac{\lambda^{n}}{n ! \mu_{1}^{n}} \frac{\lambda^{\theta-1}}{\left(n \mu_{1}\right)^{\theta-1}} \sum_{j=\theta}^{m} \frac{\lambda^{j-\theta+1}}{\prod_{i=\theta}^{j}\left(n \mu_{1}+i \mu_{2}\right)} .
\end{gathered}
$$

It follows from (8) and (9) that for $i=0,1, \ldots, n$,

$$
\pi^{\left(\boldsymbol{d}_{\theta}\right)}(i, 0)=\frac{\frac{\lambda^{i}}{i ! \mu_{1}^{i}}}{\sum_{i=0}^{n} \frac{\lambda^{i}}{i ! \mu_{1}^{i}}+\frac{\lambda^{n}}{n ! \mu_{1}^{n}} \sum_{j=1}^{\theta-1} \frac{\lambda^{j}}{\left(n \mu_{1}\right)^{j}}+\frac{\lambda^{n}}{n ! \mu_{1}^{n}} \frac{\lambda^{\theta-1}}{\left(n \mu_{1}\right)^{\theta-1}} \sum_{j=\theta}^{m} \frac{\lambda^{j-\theta+1}}{\prod_{i=\theta}^{j}\left(n \mu_{1}+i \mu_{2}\right)}} ;
$$


for $j=1,2, \ldots, \theta-1$,

$$
\pi^{\left(\boldsymbol{d}_{\theta}\right)}(n, j)=\frac{\frac{\lambda^{n}}{n ! \mu_{1}^{n}} \frac{\lambda^{j}}{\left(n \mu_{1}\right)^{j}}}{\sum_{i=0}^{n} \frac{\lambda^{i}}{i ! \mu_{1}^{i}}+\frac{\lambda^{n}}{n ! \mu_{1}^{n}} \sum_{j=1}^{\theta-1} \frac{\lambda^{j}}{\left(n \mu_{1}\right)^{j}}+\frac{\lambda^{n}}{n ! \mu_{1}^{n}} \frac{\lambda^{\theta-1}}{\left(n \mu_{1}\right)^{\theta-1}} \sum_{j=\theta}^{m} \frac{\lambda^{j-\theta+1}}{\prod_{i=\theta}^{j}\left(n \mu_{1}+i \mu_{2}\right)}} ;
$$

and for $j=\theta, \theta+1, \ldots, m$,

$$
\pi^{\left(\boldsymbol{d}_{\theta}\right)}(n, j)=\frac{\frac{\lambda^{n}}{n ! \mu_{1}^{n}} \frac{\lambda^{\theta-1}}{\left(n \mu_{1}\right)^{\theta-1}} \frac{\lambda^{j-\theta+1}}{\prod_{i=\theta}^{j}\left(n \mu_{1}+i \mu_{2}\right)}}{\sum_{i=0}^{n} \frac{\lambda^{i}}{i ! \mu_{1}^{i}}+\frac{\lambda^{n}}{n ! \mu_{1}^{n}} \sum_{j=1}^{\theta-1} \frac{\lambda^{j}}{\left(n \mu_{1}\right)^{j}}+\frac{\lambda^{n}}{n ! \mu_{1}^{n}} \frac{\lambda^{\theta-1}}{\left(n \mu_{1}\right)^{\theta-1}} \sum_{j=\theta}^{m} \frac{\lambda^{j-\theta+1}}{\prod_{i=\theta}^{j}\left(n \mu_{1}+i \mu_{2}\right)}} .
$$

It follows from (11) to (16) that for $i=0,1, \ldots, n$,

$$
f(i, 0)=R i \mu_{1}-\left(n P_{1, W}+m P_{2, S}\right) C_{1}-i C_{2}^{(1)} ;
$$

for $j=1,2, \ldots, \theta-1$,

$$
f^{\left(\boldsymbol{d}_{\theta}\right)}(n, j)=R n \mu_{1}-\left(n P_{1, W}+m P_{2, S}\right) C_{1}-\left[n C_{2}^{(1)}+j C_{2}^{(2)}\right]-n \mu_{1} C_{3}
$$

and for $j=\theta, \theta+1, \ldots, m$,

$$
\begin{aligned}
f^{\left(\boldsymbol{d}_{\theta}\right)}(n, j) & =R\left(n \mu_{1}+j \mu_{2}\right)-\left[n P_{1, W}+j P_{2, W}+(m-j) P_{2, S}\right] C_{1} \\
& -\left[n C_{2}^{(1)}+j C_{2}^{(2)}\right]-n \mu_{1} C_{3}-\lambda 1_{\{i=n, j=m\}} C_{4} .
\end{aligned}
$$

Note that

$$
\eta^{\boldsymbol{d}_{\theta}}=\sum_{i=0}^{n} \pi^{\left(\boldsymbol{d}_{\theta}\right)}(i, 0) f(i, 0)+\sum_{j=1}^{m} \pi^{\left(\boldsymbol{d}_{\theta}\right)}(n, j) f^{\left(\boldsymbol{d}_{\theta}\right)}(n, j) .
$$

We obtain the explicit expression of the long-run average profit under policy $\boldsymbol{d}_{\theta}$ as follows.

$$
\begin{aligned}
\eta^{\boldsymbol{d}_{\theta}}= & \sum_{i=0}^{n} \pi^{\left(\boldsymbol{d}_{\theta}\right)}(i, 0) f(i, 0)\left[R i \mu_{1}-\left(n P_{1, W}+m P_{2, S}\right) C_{1}-i C_{2}^{(1)}\right] \\
& +\sum_{j=1}^{\theta-1} \pi^{\left(\boldsymbol{d}_{\theta}\right)}(n, j)\left\{R n \mu_{1}-\left(n P_{1, W}+m P_{2, S}\right) C_{1}-\left[n C_{2}^{(1)}+j C_{2}^{(2)}\right]-n \mu_{1} C_{3}\right\} \\
& +\sum_{j=\theta}^{m} \pi^{\left(\boldsymbol{d}_{\theta}\right)}(n, j)\left\{R\left(n \mu_{1}+j \mu_{2}\right)-\left[n P_{1, W}+j P_{2, W}+(m-j) P_{2, S}\right] C_{1}\right. \\
& \left.-\left[n C_{2}^{(1)}+j C_{2}^{(2)}\right]-n \mu_{1} C_{3}-\lambda 1_{\{i=n, j=m\}} C_{4}\right\} .
\end{aligned}
$$


Let

$$
\theta^{*}=\underset{\theta \in\{1,2, \ldots, m+1\}}{\arg \max }\left\{\eta^{\boldsymbol{d}_{\theta}}\right\}
$$

Then, we call $\boldsymbol{d}_{\theta^{*}}$ the optimal threshold energy-efficient policy in the policy set $\mathcal{D}^{\Delta}$. Since $\mathcal{D}^{\Delta} \subset \mathcal{D}$, the partially ordered set $\mathcal{D}$ shows that $\mathcal{D}^{\Delta}$ is also a partially ordered set. Based on this, it is easy to see from the two partially ordered sets $\mathcal{D}$ and $\mathcal{D}^{\Delta}$ that

$$
\eta^{\boldsymbol{d}_{\theta^{*}}} \leq \eta^{\boldsymbol{d}^{*}}
$$

If $\eta^{\boldsymbol{d}_{\theta^{*}}}=\eta^{\boldsymbol{d}^{*}}$, then we call $\boldsymbol{d}_{\theta^{*}}$ the optimal threshold energy-efficient policy in the original policy set $\mathcal{D}$; If $\eta^{\boldsymbol{d}_{\theta^{*}}}<\eta^{\boldsymbol{d}^{*}}$, then we call $\boldsymbol{d}_{\theta^{*}}$ the suboptimal threshold energy-efficient policy in the original policy set $\mathcal{D}$.

We take a minimal positive integer $\theta^{*} \in\{1,2, \ldots, m+1\}$ such that

$$
\boldsymbol{d}_{\theta^{*}}=(0,0, \ldots, 0 ; \underbrace{0,0, \ldots, 0}_{\theta^{*}-1 \text { zeros }}, \theta^{*}, \theta^{*}+1, \ldots, m) \text {. }
$$

For the optimal threshold energy-efficient policy $\boldsymbol{d}_{\theta^{*}}$, the following theorem determines the positive or negative property of the function $G^{\left(\boldsymbol{d}_{\theta}\right)}(n, \theta)+c$ for $\theta=\theta^{*}-1, \theta^{*}, \theta^{*}+1$, although the explicit expression of the perturbation realization factor $G^{\left(\boldsymbol{d}_{\theta}\right)}(n, \theta)$ is not given yet. This may be useful for us to understand the role played by Proposition 2 in analyzing the monotonicity and optimality of the energy-efficient policies. Furthermore, we also derive the necessary condition of the optimal threshold energy-efficient policy.

Theorem 8 For the threshold energy-efficient policies of the data center, the optimal threshold policy $\boldsymbol{d}_{\theta}^{*}$ satisfies the following condition

$$
G^{\left(\boldsymbol{d}_{\theta^{*}-1}\right)}\left(n, \theta^{*}-1\right)+c \leq 0, \quad G^{\left(\boldsymbol{d}_{\theta^{*}}\right)}\left(n, \theta^{*}\right)+c \geq 0, \quad G^{\left(\boldsymbol{d}_{\theta^{*}+1}\right)}\left(n, \theta^{*}+1\right)+c \geq 0 .
$$

Proof: We consider three threshold energy-efficient policies with an interrelated structure as follows.

$$
\begin{aligned}
\boldsymbol{d}_{\theta^{*}} & =(0,0, \ldots, 0 ; \underbrace{0,0, \ldots, 0}_{\theta^{*}-1 \text { zeros }}, \theta^{*}, \theta^{*}+1, \ldots, m), \\
\boldsymbol{d}_{\theta^{*}+1} & =(0,0, \ldots, 0 ; \underbrace{0,0, \ldots, 0}_{\theta^{*}-1 \text { zeros }}, 0, \theta^{*}+1, \ldots, m), \\
\boldsymbol{d}_{\theta^{*}-1} & =(0,0, \ldots, 0 ; \underbrace{0,0, \ldots, 0}_{\theta^{*}-2 \text { zeros }}, \theta^{*}-1, \theta^{*}, \ldots, m) .
\end{aligned}
$$


It follows from Lemma 1 that for two energy-efficient policies with an interrelated structure

$$
\begin{aligned}
\boldsymbol{d} & =\left(0,0, \ldots, 0 ; d_{n, 1}, \ldots, d_{n, j-1}, \underline{d_{n, j}}, d_{n, j+1}, \ldots, d_{n, m}\right) \\
\boldsymbol{d}^{\prime} & =\left(0,0, \ldots, 0 ; d_{n, 1}, \ldots, d_{n, j-1}, \underline{d_{n, j}^{\prime}}, d_{n, j+1}, \ldots, d_{n, m}\right)
\end{aligned}
$$

it is clear that

$$
\eta^{\boldsymbol{d}^{\prime}}-\eta^{\boldsymbol{d}}=\mu_{2} \pi^{\left(\boldsymbol{d}^{\prime}\right)}\left(d_{n, j}^{\prime}-d_{n, j}\right)\left[G^{(\boldsymbol{d})}(n, j)+c\right] .
$$

Thus, we obtain

$$
\eta^{\boldsymbol{d}_{\theta^{*}+1}}-\eta^{\boldsymbol{d}_{\theta^{*}}}=-\theta^{*} \mu_{2} \pi^{\left(\boldsymbol{d}_{\theta^{*}+1}\right)}\left[G^{\left(\boldsymbol{d}_{\theta^{*}}\right)}\left(n, \theta^{*}\right)+c\right],
$$

which, together with $\eta^{\boldsymbol{d}_{\theta^{*}+1}}-\eta^{\boldsymbol{d}_{\theta^{*}}} \leq 0$, leads to

$$
G^{\left(\boldsymbol{d}_{\theta^{*}}\right)}\left(n, \theta^{*}\right)+c \geq 0
$$

Similarly, we have

$$
\eta^{\boldsymbol{d}_{\theta^{*}}}-\eta^{\boldsymbol{d}_{\theta^{*}+1}}=\theta^{*} \mu_{2} \pi^{\left(\boldsymbol{d}_{\theta^{*}}\right)}\left[G^{\left(\boldsymbol{d}_{\theta^{*}+1}\right)}\left(n, \theta^{*}+1\right)+c\right]
$$

which indicates

$$
G^{\left(\boldsymbol{d}_{\theta^{*}+1}\right)}\left(n, \theta^{*}+1\right)+c \geq 0 .
$$

Also, we have

$$
\eta^{\boldsymbol{d}_{\theta^{*}}}-\eta^{\boldsymbol{d}_{\theta^{*}-1}}=-\left(\theta^{*}-1\right) \mu_{2} \pi^{\left(\boldsymbol{d}_{\theta^{*}}\right)}\left[G^{\left(\boldsymbol{d}_{\theta^{*}-1}\right)}\left(n, \theta^{*}-1\right)+c\right]
$$

which indicates

$$
G^{\left(\boldsymbol{d}_{\theta^{*}-1}\right)}\left(n, \theta^{*}-1\right)+c \leq 0 .
$$

This completes the proof.

\section{Conclusion}

In this paper, we propose a novel dynamic decision method by applying the sensitivitybased optimization theory to study the optimal energy-efficient policy of a data center with two groups of heterogeneous servers. We propose a job transfer rule among the groupservers such that the sleep energy-efficient mechanism of Group 2 becomes more effective. To find the optimal energy-efficient policy of the data center, we set up a policy-based 
Poisson equation and provide explicit expression for its unique solution by means of the RG-factorization. Based on this, we derive the monotonicity and optimality of the long-run average profit with respect to the energy-efficient policies under some restrained service prices. We prove the optimality of the bang-bang control, which significantly reduces the action search space. We also study the threshold energy-efficient policy and derive the necessary condition of the optimal threshold policy. Different from previous works in the literature on applying the traditional MDP theory to the dynamic control of data centers, the sensitivity-based optimization method used in this paper is easier and more convenient in the study of energy-efficient data centers. This sensitivity-based optimization method may open a new avenue to study the optimal energy-efficient policy for more complicated data centers.

Along such a research line of applying the sensitivity-based optimization and the RGfactorization to the energy-efficient data centers, the extension to multiple groups of heterogeneous servers deserves further investigations. The control policy will become more complicated when multiple groups of servers are considered. Another interesting research topic is to consider different cost structures, waiting capacities, service disciplines, or job migration rules. Especially, when the job migration is not allowed in data centers, the complexity of the dynamic control problem will dramatically increase and it deserves further more investigations.

\section{Acknowledgements}

Li Xia was supported by the National Key Research and Development Program of China (2016YFB0901900, 2017YFC0704100), the National Natural Science Foundation of China under grant No. 61573206 and No. U1301254, the National 111 International Collaboration Project (B06002), and the Suzhou-Tsinghua Innovation Leading Action Project.

Quan-Lin Li was supported by the National Natural Science Foundation of China under grant No. 71671158 and No. 71471160, and by the Natural Science Foundation of Hebei province under grant No. G2017203277. 


\section{References}

[1] Barroso, L. A., Hölzle, U. (2007). The case for energy-proportional computing. Computer, Vol. 40, No. 12, 33-37.

[2] Benini, L., Bogliolo, A., De Micheli, G. (2000). A survey of design techniques for system-level dynamic power management. IEEE Transactions on Very Large Scale Integration (VLSI) Systems, Vol. 8, No. 3, 299-316.

[3] Becker, R., Zilberstein, S., Lesser, V. (2004). Decentralized Markov decision processes with event-driven interactions. In: Proceedings of the Third International Joint Conference on Autonomous Agents and Multiagent Systems, Vol. 1, 302-309.

[4] Bodenstein, C., Schryen, G., Neumann, D. (2012). Energy-aware workload management models for operation cost reduction in data centers. European Journal of Operational Research, Vol. 222, No. 1, 157-167.

[5] Cao, X. R. (2007). Stochastic learning and optimization-A sensitivity-based approach. New York: Springer.

[6] Chen, X., Wardi, Y., Yalamanchili, S. (2018). Instruction-throughput regulation in computer processors with data-center applications. Discrete Event Dynamic Systems: Theory and Applications, Vol. 28, No. 1, 127-158.

[7] De Napoli, C., Forestiero, A., Lagana, D., Lupi, G., Mastroianni, C., Spataro, L. (2016). Business Scenarios for Geographically Distributed Data Centers. RT-ICARCS-16-03.

[8] Engel, Y., Etzion, O. (2011). Towards proactive event-driven computing. In: Proceedings of the 5th ACM International Conference on Distributed Event-Based System, pp. $125-136$.

[9] Gandhi, A. (2013). Dynamic Server Provisioning for Data Center Power Management. Ph.D. Thesis, School of Computer Science, Carnegie Mellon University, Pittsburgh, USA.

[10] Gandhi, A., Doroudi, S., Harchol-Balter, M., Scheller-Wolf, A. (2014). Exact analysis of the $\mathrm{M} / \mathrm{M} / \mathrm{k} /$ setup class of Markov chains via recursive renewal reward. Queueing Systems, Vol. 77, No, 2, 177-209. 
[11] Gandhi, A., Gupta, V., Harchol-Balter, M., Kozuch, M. A. (2010). Optimality analysis of energy-performance trade-off for server farm management. Performance Evaluation, Vol. 67, No, 11, 1155-1171.

[12] Gandhi, A., Harchol-Balter, M. (2013). M/G/k with staggered setup. Operations Research Letters, Vol. 41, No. 4, 317-320.

[13] Gandhi, A., Harchol-Balter, M., Adan, I. (2010). Server farms with setup costs. Performance Evaluation, Vol. 67, No. 11, 1123-1138.

[14] Gandhi, A., Harchol-Balter, M., Kozuch, M. A. (2012). Are sleep states effective in data centers? In: 2012 International Green Computing Conference (IGCC), pp. 1-10.

[15] Gebrehiwot, M. E., Aalto, S., Lassila, P. (2016). Optimal energy-aware control policies for FIFO servers. Performance Evaluation, Vol. 103, 41-59.

[16] Gebrehiwot, M. E., Aalto, S., Lassila, P. (2016). Energy-performance trade-off for processor sharing queues with setup delay. Operations Research Letters, Vol. 44, No. 1, 101-106.

[17] Gebrehiwot, M. E., Aalto, S., Lassila, P. (2017). Energy-aware SRPT server with batch arrivals: Analysis and optimization. Performance Evaluation, Vol. 115, 92-107.

[18] Hassin, R., Shaki, Y. Y., Yovel, U. (2015). Optimal service-capacity allocation in a loss system. Naval Research Logistics, Vol. 62, No. 2, 81-97.

[19] Hipp, S. K., Holzbaur, U. D. (1988). Decision processes with monotone hysteretic policies. Operations Research, Vol. 36, No. 4, 585-588.

[20] Huang, L., Neely, M. J. (2013). Utility optimal scheduling in energy-harvesting networks. IEEE/ACM Transactions on Networking, Vol. 21, No. 4, 1117-1130.

[21] Hong, K. S., Lee, C. (2013). Integrated pricing and capacity decision for a telecommunication service provider. Multimedia Tools and Applications, Vol. 64, No. 2, 389-406.

[22] Hunter, J. J. (1982). Generalized inverses and their application to applied probability problems. Linear Algebra and its Applications, Vol. 45, 157-198. 
[23] Kamitsos, I., Andrew, L., Kim, H., Chiang, M. (2010). Optimal sleep patterns for serving delay-tolerant jobs. In: Poceedings of the 1st International Conference on Energy-Efficient Computing and Networking, pp. 31-40.

[24] Kamitsos, I., Andrew, L., Kim, H., Ha, S. (2012). Better energy-delay tradeoff via server resource pooling. In: The 2012 International Conference on Computing, Networking and Communications, pp. 611-616.

[25] Kamitsos, I., Ha, S., Andrew, L., Bawa, J., Butnariu, D., Kim, H., Chiang, M. (2017). Optimal sleeping: models and experiments for energy-delay tradeoff. International Journal of Systems Science: Operations \& Logistics, Vol. 4, No. 4, 356-371.

[26] Kliazovich, D., Bouvry, P., Khan, S. U. (2012). GreenCloud: a packet-level simulator of energy-aware cloud computing data centers. The Journal of Supercomputing, Vol. 62, No. 3, 1263-1283.

[27] Koole, G. (1998). Structural results for the control of queueing systems using eventbased dynamic programming. Queueing Systems, Vol. 30, No. 3-4, 323-339.

[28] Kuehn, P. J., Mashaly, M. E. (2015). Automatic energy efficiency management of data center resources by load-dependent server activation and sleep modes. Ad Hoc Networks, Vol. 25, Part B, 497-504.

[29] Li, Q. L. (2010). Constructive computation in stochastic models with applications: the $R G$-factorizations. Springer.

[30] Li, Q. L., Cao, J. (2004). Two types of RG-factorizations of quasi-birth-and-death processes and their applications to stochastic integral functionals. Stochastic Models, Vol. 20, No. 3, 299-340.

[31] Li, Q. L., Ma, J. Y., Xie, M. Z., Xia, L. (2017). Group-server queues. In: International Conference on Queueing Theory and Network Applications, pp. 49-72.

[32] Lu, F. V., Serfozo, R. F. (1984). M/M/1 queueing decision processes with monotone hysteretic optimal policies. Operations Research, Vol. 32, No. 5, 1116-1132.

[33] Maccio, V. J., Down, D. G. (2015). On optimal policies for energy-aware servers. Performance Evaluation, Vol. 90, 36-52. 
[34] Mazzucco, M., Dyachuk, D., Deters, R. (2010). Maximizing cloud providers revenues via energy aware allocation policies. In: IEEE International Conference on Cloud Computing, pp. 131-138.

[35] Mitrani, I. (2011). Service center trade-offs between customer impatience and power consumption. Performance Evaluation, Vol. 68, No. 11, 1222-1231.

[36] Mitrani, I. (2013). Managing performance and power consumption in a server farm. Annals of Operations Research, Vol. 202, No. 1, 121-134.

[37] Natural Resources Defense Council. (2016). Environmental Issues [Online]. Available: https://www.nrdc.org/resources/americas-data-centers-consuming-and-wastinggrowing-amounts-energy.

[38] Phung-Duc, T. (2017). Exact solutions for M/M/c/setup queues. Telecommunication Systems, Vol. 64, No. 2, 309-324.

[39] Puterman, M. L. (2014). Markov decision processes: discrete stochastic dynamic programming. John Wiley \& Sons.

[40] Qiu, Q., Pedram, M. (1999). Dynamic power management based on continuous-time Markov decision processes. In: Proceedings of the 36th Annual ACM/IEEE Design Automation Conference, pp. 555-561.

[41] Qiu, Q., Qu, Q., Pedram, M. (2001). Stochastic modeling of a power-managed systemconstruction and optimization. IEEE Transactions on Computer-Aided Design of Integrated Circuits and Systems, Vol. 20, No. 10, 1200-1217.

[42] Schwartz, C., Pries, R., Tran-Gia, P. (2012). A queuing analysis of an energy-saving mechanism in data centers. In: The International Conference on Information Network (ICOIN), pp. 70-75.

[43] Shehabi, A., Smith, S., Sartor, D., et al. (2016). United States Data Center Energy Usage Report. Lawrence Berkely Lab.

[44] Šimunić, T., Benini, L., Glynn, P., De Micheli, G. (2001). Event-driven power management. IEEE Transactions on Computer-Aided Design of Integrated Circuits and Systems, Vol. 20, No. 7, 840-857. 
[45] Tan, Y., Lu, Y., Xia, C. H. (2012). Provisioning for large scale loss network systems with applications in cloud computing. ACM Sigmetrics Performance Evaluation Review, Vol. 40, No. 3, 83-85.

[46] Xia, L. (2014). Service rate control of closed Jackson networks from game theoretic perspective. European Journal of Operational Research, Vol. 237, No. 2, 546-554.

[47] Xia, L. (2014). Event-based optimization of admission control in open queueing networks. Discrete Event Dynamic Systems: Theory and Applications, Vol. 24, No. 2, $133-151$.

[48] Xia, L., Cao, X. R. (2012). Performance optimization of queueing systems with perturbation realization. European Journal of Operational Research, Vol. 218, No. 2, 293304.

[49] Xia, L., Chen, S. (2018). Dynamic pricing control for open queueing networks. IEEE Transactions on Automatic Control, Online Publication, Pages 1-11.

[50] Xia, L., He, Q. M., Alfa, A. S. (2017). Optimal control of state-dependent service rates in a MAP/M/1 queue. IEEE Transactions on Automatic Control, Vol. 62, No. $10,4965-4979$.

[51] Xia, L., Jia, Q. S. (2015). Parameterized Markov decision process and its application to service rate control. Automatica, Vol. 54, 29-35.

[52] Xia, L., Jia, Q. S., Cao, X. R. (2014). A tutorial on event-based optimization-A new optimization framework. Discrete Event Dynamic Systems: Theory and Applications, Vol. 24, No. 2, 103-132.

[53] Xia, L., Miller, D., Zhou, Z., Bambos, N. (2017). Service rate control of tandem queues with power constraints. IEEE Transactions on Automatic Control, Vol. 62, No. $10,5111-5123$.

[54] Xia, L., Shihada, B. (2013). Max-min optimality of service rate control in closed queueing networks. IEEE Transactions on Automatic Control, Vol. 58, No. 4, 10511056. 
[55] Xia, L., Shihada, B. (2015). A Jackson network model and threshold policy for joint optimization of energy and delay in multi-hop wireless networks. European Journal of Operational Research, Vol. 242, No. 3, 778-787.

[56] Yang, J., Zhang, S., Wu, X., Ran, Y., Xi, H. (2017). Online learning-based server provisioning for electricity cost reduction in data center. IEEE Transactions on Control Systems Technology, Vol. 25, No. 3, 1044-1051.

[57] Yao, Y., Huang, L., Sharma, A. B., Golubchik, L., Neely, M. J. (2014). Power cost reduction in distributed data centers: A two-time-scale approach for delay tolerant workloads. IEEE Transactions on Parallel and Distributed Systems, Vol. 25, No. 1, 200-211. 